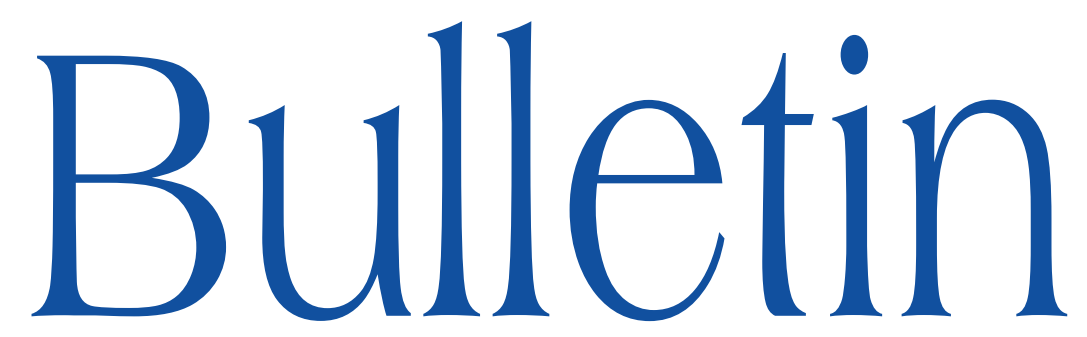

de la SOCIÉTÉ MATHÉMATIQUE DE FRANCE

\title{
SÉRIES HYPERGÉOMÉTRIQUES MULTIPLES ET POLYZÊTAS
}

J. Cresson \& S. Fischler \& T. Rivoal

\section{Tome 136} Fascicule 1 


\title{
SÉRIES HYPERGÉOMÉTRIQUES MULTIPLES ET POLYZÊTAS
}

\author{
par J. Cresson, S. Fischler \& T. Rivoal
}

\begin{abstract}
RÉSumÉ. - Nous décrivons un algorithme théorique et effectif permettant de démontrer que des séries et intégrales hypergéométriques multiples relativement générales se décomposent en combinaisons linéaires à coefficients rationnels de polyzêtas.
\end{abstract}

Abstract (Multiple hypergeometric series and polyzetas). - We describe a theoretical and effective algorithm which enables us to prove that rather general hypergeometric series and integrals can be decomposed as linear combinations of multiple zeta values, with rational coefficients.

\section{Introduction}

Une généralisation de la fonction zêta de Riemann $\zeta(s)$ est donnée par les séries polyzêtas, définies pour tout entier $p \geq 1$ et tout $p$-uplet $\underline{s}=\left(s_{1}, s_{2}, \ldots, s_{p}\right)$

Texte reçu le 12 septembre 2006, accepté le 15 décembre 2006

J. Cresson, Laboratoire de Mathématiques appliquées de Pau, Bâtiment I.P.R.A, Université de Pau et des Pays de l'Adour, av. de l'Université, BP 1155, 64013 Pau Cedex (France).

S. Fischler, Université Paris-Sud, Laboratoire de Mathématiques, UMR CNRS 8628, Bât. 425, 91405 Orsay Cedex (France).

T. Rivoal, Institut Fourier, CNRS UMR 5582, Université Grenoble 1, 100 rue des Maths, BP 74, 38402 Saint-Martin d'Hères Cedex (France).

Classification mathématique par sujets (2000). - 33C70, 11M41, 11M06, 11J72.

Mots clefs. - Polyzêta, série hypergéométrique multiple, algorithme. 
d'entiers $\geq 1$, avec $s_{1} \geq 2$, par

$$
\zeta\left(s_{1}, s_{2}, \ldots, s_{p}\right)=\sum_{k_{1}>k_{2}>\cdots>k_{p} \geq 1} \frac{1}{k_{1}^{s_{1}} k_{2}^{s_{2}} \cdots k_{p}^{s_{p}}} .
$$

Les entiers $p$ et $s_{1}+s_{2}+\cdots+s_{p}$ sont respectivement la profondeur et le poids de $\zeta\left(s_{1}, s_{2}, \ldots, s_{p}\right)$; pour $p=0$, on pose

$$
\zeta(\varnothing)=1 .
$$

Pour diverses raisons, il est plus simple de considérer que la sommation est faite sur $k_{1} \geq k_{2} \geq \cdots \geq k_{p} \geq 1$ : nous noterons $\bar{\zeta}\left(s_{1}, s_{2}, \ldots, s_{p}\right)$ les séries ainsi obtenues. Il est à noter que les deux séries convergent plus généralement pour des exposants complexes vérifiant $\sum_{j=1}^{r} \operatorname{Re}\left(s_{j}\right)>r$ pour tout $r \in\{1, \ldots, p\}$, ce qui autorise à avoir des exposants entiers négatifs par exemple.

Les polyzêtas interviendront dans cet article par l'intermédiaire des fonctions polylogarithmes multiples, définies pour $\left|z_{1}\right| \leq 1, \ldots,\left|z_{p}\right| \leq 1$ par

$$
\operatorname{Li}_{s_{1}, s_{2} \ldots, s_{p}}\left(z_{1}, z_{2}, \ldots, z_{p}\right)=\sum_{k_{1}>k_{2}>\cdots>k_{p} \geq 1} \frac{z_{1}^{k_{1}} z_{2}^{k_{2}} \cdots z_{p}^{k_{p}}}{k_{1}^{s_{1}} k_{2}^{s_{2}} \cdots k_{p}^{s_{p}}} .
$$

On obtiendra en fait les résultats pour les polylogarithmes multiples larges, définis par

$$
\operatorname{La}_{s_{1}, s_{2} \ldots, s_{p}}\left(z_{1}, z_{2} \ldots, z_{p}\right)=\sum_{k_{1} \geq k_{2} \geq \cdots \geq k_{p} \geq 1} \frac{z_{1}^{k_{1}} z_{2}^{k_{2}} \cdots z_{p}^{k_{p}}}{k_{1}^{s_{1}} k_{2}^{s_{2}} \cdots k_{p}^{s_{p}}}
$$

et notés «Le » par Ulanskiı̌ et Zlobin. Lorsque $p=1$, les deux variantes coïncident avec les polylogarithmes usuels et si $z_{1}=z_{2}=\cdots=z_{p}=1$ et $s_{1} \geq 2$, on a

$$
\begin{aligned}
\operatorname{Li}_{s_{1}, s_{2} \ldots, s_{p}}(1,1, \ldots, 1) & =\zeta\left(s_{1}, s_{2} \ldots, s_{p}\right), \\
\operatorname{La}_{s_{1}, s_{2} \ldots, s_{p}}(1,1, \ldots, 1) & =\bar{\zeta}\left(s_{1}, s_{2} \ldots, s_{p}\right) .
\end{aligned}
$$

Un théorème d'Ulanskiı̌ [40] permet de passer linéairement d'un type de série à l'autre; en vue d'applications diophantiennes, on ne perd donc rien à considérer une variante plutôt qu'une autre.

Remarquons dès à présent que les fonctions polylogarithmes multiples peuvent être définies pour des exposants $s_{i}$ complexes, à condition de supposer en plus que $\left|z_{1}\right|<1$ pour des raisons de convergence. En particulier, nous utiliserons ces fonctions avec des $s_{j} \in \mathbb{Z}:$ par définition, le poids d'une telle fonction est alors $\sum_{j=1}^{p} \max \left(s_{j}, 0\right)$.

On voit naturellement apparaître les polyzêtas lorsque, par exemple, on considère les produits des valeurs de la fonction zêta : on a

$$
\zeta(n) \zeta(m)=\zeta(n+m)+\zeta(n, m)+\zeta(m, n)
$$

TOME $136-2008-\mathrm{N}^{\mathrm{O}} 1$ 
ce qui permet en quelque sorte de «linéariser » ces produits. En dehors de quelques identités telles que $\zeta(2,1)=\zeta(3)$ (due à Euler), la nature arithmétique de ces séries est aussi peu connue que celle des nombres $\zeta(s)$. Cependant, l'ensemble des nombres $\zeta(\underline{s})$ possède une très riche structure algébrique assez bien comprise, au moins conjecturalement (voir [42]). Par exemple, on peut s'intéresser aux $\mathbb{Q}$-sous-espaces vectoriels $\mathcal{Z}_{p}$ de $\mathbb{R}$, engendrés par les $2^{p-2}$ polyzêtas de poids $p \geq 2$ :

$$
\begin{aligned}
& \mathcal{Z}_{2}=\mathbb{Q} \zeta(2), \\
& \mathcal{Z}_{3}=\mathbb{Q} \zeta(3)+\mathbb{Q} \zeta(2,1), \\
& \mathcal{Z}_{4}=\mathbb{Q} \zeta(4)+\mathbb{Q} \zeta(3,1)+\mathbb{Q} \zeta(2,2)+\mathbb{Q} \zeta(2,1,1), \text { etc. }
\end{aligned}
$$

Posons $v_{p}=\operatorname{dim}_{\mathbb{Q}}\left(\mathcal{Z}_{p}\right)$. On a alors la

Conjecture 1.1. - (i) Pour tout entier $p \geq 2$, on a $v_{p}=c_{p}$, où l'entier $c_{p}$ est défini par la récurrence de type Fibonacci

$$
c_{p+3}=c_{p+1}+c_{p}, \quad \text { avec } \quad c_{0}=1, c_{1}=0, c_{2}=1 .
$$

(ii) Les $\mathbb{Q}$-espaces vectoriels $\mathbb{Q}$ et $\mathcal{Z}_{p}(p \geq 2)$, sont en somme directe.

La suite $\left(v_{p}\right)_{p \geq 2}$ devrait donc croître comme $\alpha^{p}$ (où $\alpha \approx 1,3247$ est racine du polynôme $\left.X^{3}-X-1\right)$, ce qui est bien plus petit que $2^{p-2}$. Il y a donc conjecturalement beaucoup de relations linéaires entre les polyzêtas de même poids et aucune en poids différents : dans cette direction, un théorème de Goncharov [19] et Terasoma [39] affirme que l'on a $v_{p} \leq c_{p}$ pour tout entier $p \geq 2$. Il reste donc à montrer l'inégalité inverse pour montrer (i) mais aucune minoration non triviale de $v_{p}$ n'est connue à ce jour : si l'on montre facilement que $v_{2}=v_{3}=v_{4}=1$, on est bloqué dès l'égalité $v_{5}=2$, qui est équivalente à l'irrationalité toujours inconnue de $\zeta(5) /(\zeta(3) \zeta(2))$. Plus généralement, un des intérêts de la conjecture 1.1 est d'impliquer la suivante.

Conjecture 1.2. - Les nombres $\pi, \zeta(3), \zeta(5), \zeta(7), \zeta(9)$, etc. sont algébriquement indépendants sur $\mathbb{Q}$.

Cette conjecture semble actuellement totalement hors de portée. Un certain nombre de résultats diophantiens ont néanmoins été obtenus en profondeur 1 , c'est-à-dire dans le cas de la fonction zêta de Riemann :

(i) Le nombre $\zeta(3)$ est irrationnel (Apéry [3]);

(ii) La dimension de l'espace vectoriel engendré sur $\mathbb{Q}$ par $1, \zeta(3), \zeta(5), \ldots$, $\zeta(A)$ (avec $A$ impair) croît au moins comme $\log (A)$ (cf. [5], [33]); 
(iii) Au moins un des quatre nombres $\zeta(5), \zeta(7), \zeta(9), \zeta(11)$ est irrationnel (Zudilin [49]).

Ces résultats peuvent être obtenus par l'étude de certaines séries de la forme ${ }^{(1)}$

$$
\sum_{k=1}^{\infty} \frac{P(k)}{k^{A}(k+1)^{A} \cdots(k+n)^{A}} z^{-k}
$$

avec $P(X) \in \mathbb{Q}[X], n \geq 0, A \geq 1$ et $|z| \geq 1$ (le choix de $z^{-1}$ plutôt que $z$ est purement technique) : nous rappelons sommairement au paragraphe 3.1 comment on utilise ces séries pour les démontrer, en exploitant le fait que, génériquement, elles s'expriment aussi comme combinaisons linéaires des valeurs de zêta aux entiers lorsque $z=1$. Les divers choix de $P$ conduisent à des séries hypergéométriques généralisées : voir les ouvrages [4], [34] pour les définitions, qui ne sont pas essentielles ici.

Notre but est de poser les bases d'une généralisation de cette méthode hypergéométrique en profondeur quelconque en considérant a priori des séries multiples de la forme

$$
\sum_{k_{1} \geq \cdots \geq k_{p} \geq 1} \frac{P\left(k_{1}, \ldots, k_{p}\right)}{\left(k_{1}\right)_{n_{1}+1}^{A_{1}} \cdots\left(k_{p}\right)_{n_{p}+1}^{A_{p}}} z_{1}^{-k_{1}} \cdots z_{p}^{-k_{p}},
$$

avec $P\left(X_{1}, \ldots, X_{p}\right) \in \mathbb{Q}\left[X_{1}, \ldots, X_{p}\right]$, des entiers $A_{j} \geq 1$ et $n_{j} \geq 0$ et $\left|z_{1}\right| \geq 1, \ldots,\left|z_{p}\right| \geq 1$, ceci dans l'espoir qu'elles s'expriment comme combinaisons linéaires de polyzêtas intéressants lorsque $z_{1}=\cdots=z_{p}=1$. Pour raccourcir les expressions, on a utilisé le symbole de Pochhammer

$$
(\alpha)_{m}=\alpha(\alpha+1) \cdots(\alpha+m-1) .
$$

On pourrait imaginer généraliser encore (1.2) en remplaçant, au dénominateur, chaque facteur $\left(k_{i}\right)_{n_{i}+1}^{A_{i}} \operatorname{par}\left(k_{i}+r_{i}\right)_{n_{i}+1}^{A_{i}}$. Cela peut être utile (et nos méthodes le permettent) si des bornes explicites apparaissent en fonction des $n_{i}$, mais c'est inutile pour des résultats qualitatifs car on peut s'y ramener, en remplaçant $n_{i}$ par $n_{i}+r_{i}$ et en multipliant le numérateur par $\left(k_{i}\right)_{r_{i}}^{A_{i}}$.

Les séries de la forme (1.2) apparaissent naturellement dans la littérature. Par exemple, Sorokin [36] a déduit l'irrationalité de $\zeta(3)$ d'un résultat que l'on peut écrire ainsi (voir $\S 2.2$ ) : pour tout entier $n \geq 0$, on a

$$
n ! \sum_{k_{1} \geq k_{2} \geq 1} \frac{\left(k_{2}-n\right)_{n}\left(k_{1}-k_{2}+1\right)_{n}}{\left(k_{1}\right)_{n+1}^{2}\left(k_{2}\right)_{n+1}}=2 a_{n} \zeta(2,1)-b_{n}
$$

(1) Du moins, dans le cas de (i) et (ii); le point (iii) nécessite une idée a priori différente (série « dérivée ») mais on peut l'intégrer dans le cadre fourni par (1.1). Voir un peu plus loin dans cette introduction pour plus de détails.

TOME $136-2008-\mathrm{N}^{\mathrm{O}} 1$ 
où $a_{n}$ et $b_{n}$ sont les célèbres nombres rationnels utilisés par Apéry [3] dans sa preuve originelle de l'irrationalité de $\zeta(3)$. La méthode de Sorokin n'utilise pas directement la série (1.3) mais consiste à résoudre un problème subtil d'approximation de Padé, qu'il n'est malheureusement pas facile de généraliser à d'autres situations. Nous nous affranchissons de l'approximation de Padé pour espérer profiter, en profondeur supérieure, de la grande souplesse de la méthode hypergéométrique en profondeur 1 . Il est intéressant de noter que la série double en (1.3) est un exemple de série hypergéométrique de Kampé de Fériet (voir [38, p. 27]), comme on le voit après quelques transformations triviales du sommande. Par un léger abus de langage, nous appelons série hypergéométrique multiple une expression de la forme (1.2) bien que, en général, il ne s'agisse seulement que de combinaisons linéaires rationnelles de telles séries.

Un ingrédient, fréquemment utilisé avec des séries simples, consiste à dériver la fraction rationnelle en $k$ dans la série (1.1), avant de sommer ; par exemple, une double dérivation sert à démontrer le résultat de Zudilin [49] rappelé après la conjecture 1.2. Cette astuce, appliquée plusieurs fois, permet de faire disparaître $\zeta(s)$ de la forme linéaire obtenue, pour de petites valeurs de $s$. On peut imaginer l'utiliser pour des sommes multiples, même si on n'a aucun résultat connu de disparition de polyzêtas dans ce cadre (voir cependant [15]). Il est clair qu'en dérivant une fraction rationnelle de la forme

$$
\frac{P\left(X_{1}, \ldots, X_{p}\right)}{\left(X_{1}\right)_{n+1}^{A_{1}} \ldots\left(X_{p}\right)_{n+1}^{A_{p}}}
$$

par rapport à l'une des variables $X_{j}$, on obtient une fraction rationnelle de la même forme (avec $A_{j}$ remplacé par $A_{j}+1$ ) : cette remarque montre que l'on ne perd rien à considérer des séries de la forme (1.2).

En profondeur $p \geq 2$, l'étude des séries multiples du type de (1.2) se décompose en plusieurs étapes et, malheureusement, la première difficulté se présente dès la première étape, qui est pourtant triviale en profondeur 1 . Nous mettons ceci en évidence sur l'exemple de la profondeur 2 au paragraphe 3.2 : la généralisation en profondeur quelconque nécessite la production d'un algorithme récursif (permettant de déduire le cas de la profondeur $p$ du cas de la profondeur $p-1$ ) que l'on décrit au paragraphe 4.3. Informellement, on obtient alors le résultat suivant.

THÉORÈme 1.3. - Supposons que l'on ait $\left|z_{1}\right|>1$ et $\left|z_{j}\right| \geq 1$ pour tout $j=$ $2, \ldots, p$. Alors, toute série de la forme (1.2) s'écrit comme une combinaison linéaire à coefficients polynômes de Laurent dans $\mathbb{Q}\left[z_{1}^{ \pm 1}, \ldots, z_{p}^{ \pm 1}\right]$ en les polylogarithmes multiples $\operatorname{La}_{s_{1}, \ldots, s_{q}}\left(1 / \widehat{z}_{1}, \ldots, 1 / \widehat{z}_{q}\right)$ où $0 \leq q \leq p, \sum_{j=1}^{q} \max \left(s_{j}, 0\right) \leq$ $\sum_{j=1}^{p} A_{j}$ et où les $\widehat{z}_{1}, \ldots, \widehat{z}_{q}$ sont certains produits des $z_{1}, \ldots, z_{p}$. 
Remarque 1. - 1) Bien que peu surprenant en apparence, ce résultat est, comme on le verra, loin d'être facile à démontrer. Ici, les entiers $s_{1}, \ldots, s_{q}$ peuvent être de signe quelconque et, comme dans toute la suite, on doit entendre un polylogarithme de profondeur 0 comme étant la fonction identiquement égale à 1 .

2) Certains des $s_{j}$ peuvent être négatifs ou nuls : cela ne peut être le cas que si l'un des degrés en l'une des variables $X_{j}$ de la fraction

$$
\frac{P\left(X_{1}, \ldots, X_{p}\right)}{\left(X_{1}\right)_{n+1}^{A_{1}} \ldots\left(X_{p}\right)_{n+1}^{A_{p}}}
$$

est positif, c'est-à-dire lorsque $\operatorname{deg}_{X_{j}}(P) \geq A_{j}\left(n_{j}+1\right)$.

3) On peut raffiner ce théorème : voir le théorème 5.1 au paragraphe 5 . Il en résulte par exemple que les polynômes de Laurent sont en fait toujours dans $\mathbb{Q}\left[z_{1}, z_{2}^{ \pm 1}, \ldots, z_{p}^{ \pm 1}\right]$.

Une deuxième difficulté provient du fait que certains polylogarithmes multiples peuvent avoir un ou des exposants $s_{j} \leq 0$, ce qui nécessite un traitement à part. On obtient le résultat dit de non-enrichissement suivant (voir le paragraphe 6) :

Lorsque les modules de $z_{1}, \ldots, z_{p}$ sont tous différents de 1 , tout polylogarithme multiple $\mathrm{La}_{s_{1}, \ldots, s_{p}}(\underline{z})$, de profondeur $p$ et ayant certains exposants $\leq 0$, est une combinaison linéaire en des polylogarithmes multiples d'indices $\geq 1$ (en des produits des $\left.z_{j}\right)$ de poids $\leq \sum_{j=1}^{p} \max \left(s_{j}, 0\right)$, dont les coefficients sont des polynômes à coefficients rationnels en les

$$
\left(\left(1-z_{j_{1}} \cdots z_{j_{m}}\right)^{-1}\right)_{1 \leq j_{1}<\cdots<j_{m} \leq p, m \geq 1} \text { et les } \quad\left(z_{j}^{ \pm 1}\right)_{1 \leq j \leq p} .
$$

En combinant ce résultat et le théorème 1.3 on obtient l'énoncé suivant (qui a été obtenu indépendamment, dans le cas particulier $z_{1}=\cdots=z_{p}$, par Zlobin [46]) :

THÉORÈme 1.4. - Supposons que l'on ait $\left|z_{j}\right|>1$ pour tout $j=1, \ldots, p$. Alors, toute série de la forme (1.2) s'écrit comme une combinaison linéaire à coefficients polynômes à coefficients rationnels en les

$$
\left(\left(1-z_{j_{1}} \cdots z_{j_{m}}\right)^{-1}\right)_{1 \leq j_{1}<\cdots<j_{m} \leq p, m \geq 1} \text { et les }\left(z_{j}^{ \pm 1}\right)_{1 \leq j \leq p}
$$

de polylogarithmes multiples $\operatorname{La}_{s_{1}, \ldots, s_{q}}\left(1 / \widehat{z}_{1}, \ldots, 1 / \widehat{z}_{q}\right)$ où $0 \leq q \leq p, s_{i} \geq 1$ pour $i=1, \ldots, q, \sum_{j=1}^{q} s_{j} \leq \sum_{j=1}^{p} A_{j}$ et où les $\widehat{z}_{1}, \ldots, \widehat{z}_{q}$ sont certains produits des $z_{1}, \ldots, z_{p}$. 
L'analogue des théorèmes 1.3 et 1.4 lorsque $z_{1}=\cdots=z_{p}=1$ s'énonce comme suit. Une version plus précise (le théorème 7.2) sera démontrée au paragraphe 7.4 ; la démonstration nécessite d'utiliser la régularisation des polyzêtas divergents. Ce théorème est celui que nous avons implémenté dans [11], ce qui nous a permis d'avoir l'idée du théorème 1.6 ci-dessous et d'observer d'autres exemples de séries qui font apparaître seulement certains des polyzêtas attendus [15].

THÉORÈme 1.5. - Toute série convergente de la forme (1.2) s'écrit, lorsque $z_{1}=\cdots=z_{p}=1$, comme une combinaison linéaire à coefficients rationnels en les polyzêtas $\zeta\left(s_{1}, \ldots, s_{q}\right)$ où $0 \leq q \leq p, s_{1} \geq 2, s_{i} \geq 1$ pour $i=1, \ldots, q$ et $\sum_{j=1}^{q} s_{j} \leq \sum_{j=1}^{p} A_{j}$.

Notre algorithme donne diverses précisions sur les théorèmes $1.3,1.4$ et 1.5 (dénominateurs des coefficients, degré des polynômes dans le cas des séries les plus simples, dites briques). De plus, il se prête (pour tous $z_{1}, \ldots, z_{p}$ ) à une implémentation informatique que nous avons effectuée [11] (lorsque $z_{1}=\cdots=$ $z_{p}=1$ ) à l'aide du programme GP/Pari : cela nous a permis de tester de nombreuses séries et d'obtenir des résultats tels que

$$
\begin{aligned}
\sum_{k_{1} \geq k_{2} \geq 1} & \frac{5 k_{2}^{2}-k_{1}^{2}-4 k_{1} k_{2}-3 k_{1}+7 k_{2}}{\left(k_{1}\right)_{3}^{4}\left(k_{2}+1\right)_{4}^{3}} \\
=- & \frac{153060027667}{1289945088}+\frac{832127737}{17915904} \zeta(2)+\frac{33349589}{2985984} \zeta(3) \\
+ & \frac{10561397}{2985984} \zeta(4)+\frac{117277}{10368} \zeta(5)+\frac{1475}{1728} \zeta(6)+\frac{757}{432} \zeta(7) \\
& +\frac{6125}{1728} \zeta(2,2)+\frac{245}{24} \zeta(2,3)+\frac{35}{32} \zeta(3,2) \\
& +\frac{1}{6} \zeta(3,3)+\frac{595}{864} \zeta(4,2)+\frac{7}{4} \zeta(4,3) .
\end{aligned}
$$

Ce résultat pourrait éventuellement être un peu simplifié en utilisant les relations linéaires connues entre polyzêtas.

Cette étape franchie, une troisième difficulté provient de la profusion de polyzêtas qui semblent apparaître spontanément dans des exemples « au hasard » comme (1.4). Nous avons donc été conduits à rechercher une classe de polynômes $P\left(X_{1}, \ldots, X_{p}\right)$ tels que, a priori, seulement certains polyzêtas intéressants ont un coefficient non nul à la sortie de l'algorithme. Par «intéressants », nous entendons des polyzêtas qui ne sont pas trivialement des puissances de $\pi$, 
qui parasitent les applications diophantiennes en les rendant triviales ${ }^{(2)}$. Voici quelques exemples de séries qui ne font pas apparaître $\pi$ :

$$
\begin{gathered}
\sum_{k_{1} \geq k_{2} \geq 1}\left(k_{1}+1\right)\left(k_{2}+1\right) \frac{\left(k_{1}-k_{2}-1\right)_{3}\left(k_{1}+k_{2}+1\right)_{3}\left(k_{1}-1\right)_{5}\left(k_{2}-1\right)_{5}}{\left(k_{1}\right)_{3}^{5}\left(k_{2}\right)_{3}^{5}} \\
=\frac{27875}{8192}-\frac{2847}{1024} \zeta(3)-\frac{15}{32} \zeta(5)+\frac{27}{64} \zeta(7), \\
\sum_{k_{1} \geq k_{2} \geq 1}\left(k_{1}+\frac{1}{2}\right)\left(k_{2}+\frac{1}{2}\right) \frac{\left(k_{1}-k_{2}-1\right)_{3}\left(k_{1}+k_{2}\right)_{3}\left(k_{1}-1\right)_{4}\left(k_{2}-1\right)_{4}}{\left(k_{1}\right)_{2}^{7}\left(k_{2}\right)_{2}^{7}} \\
=-1156+891 \zeta(3)+\frac{189}{2} \zeta(5)+78(\zeta(5,3)-\zeta(3,5)) \\
\sum_{k_{1} \geq k_{2} \geq 1} \frac{\left(k_{1}-k_{2}\right)\left(k_{1}+k_{2}+4\right)\left(k_{1}-2\right)_{9}\left(k_{2}-2\right)_{9}^{4}\left(k_{2}\right)_{5}^{4}}{\left(k_{1}\right.} \\
=-\frac{642739948033}{41278242816}+\frac{10214719}{995328} \zeta(3)+\frac{57497}{18432} \zeta(5), \\
\sum_{k_{1} \geq k_{2} \geq 3 \geq 1}^{\left(k_{1}+\frac{1}{2}\right)\left(k_{2}+\frac{1}{2}\right)\left(k_{3}+\frac{1}{2}\right)} \\
\times \frac{\left(k_{1}-k_{2}\right)\left(k_{2}-k_{3}\right)\left(k_{1}-k_{3}\right)\left(k_{1}+k_{2}+1\right)\left(k_{1}+k_{3}+1\right)\left(k_{2}+k_{3}+1\right)}{\left(k_{1}\right)_{2}^{4}\left(k_{2}\right)_{2}^{4}\left(k_{3}\right)_{2}^{4}} \\
=-\frac{1}{4}-\zeta(3)+\frac{1}{4} \zeta(5)+\zeta(3)^{2}-\frac{1}{4} \zeta(7) .
\end{gathered}
$$

Nous avons proposé dans [12] une généralisation en profondeur quelconque des séries very-well-poised ${ }^{(3)}$ (ou très bien équilibrées) introduites en profondeur 1 ; elle explique les quatre exemples ci-dessus. Il s'agit du résultat suivant, qui est démontré sous une forme plus précise dans [12].

THÉORÈme 1.6. - Fixons trois entiers $A \geq 2, n \geq 0$ et $p \geq 1$, ainsi que $P\left(X_{1}, \ldots, X_{p}\right) \in \mathbb{Q}\left[X_{1}, \ldots, X_{p}\right]$ un polynôme tel que

$$
P\left(X_{\sigma(1)}, X_{\sigma(2)}, \ldots, X_{\sigma(p)}\right)=\varepsilon(\sigma) P\left(X_{1}, X_{2}, \ldots, X_{p}\right)
$$

(2) Par exemple, la minoration de la dimension de l'espace des nombres $\zeta(2 n+1)$ devient sans intérêt lorsque l'on rajoute les nombres $\zeta(2 n)$ : la transcendance de $\pi$ implique leur indépendance linéaire sur $\mathbb{Q}$ et donc une minoration de dimension de l'ordre de $\frac{1}{2} A$ au lieu de $\log (A)$.

(3) Voir le paragraphe 3.1 pour l'origine de cette dénomination. 
pour tout $\sigma \in \mathfrak{S}_{p}($ où $\varepsilon(\sigma)$ désigne la signature de $\sigma)$, et

$$
\begin{aligned}
& P\left(X_{1}, \ldots, X_{j-1},-X_{j}-n, X_{j+1}, \ldots, X_{p}\right) \\
& \quad=(-1)^{A(n+1)+1} P\left(X_{1}, \ldots, X_{j-1}, X_{j}, X_{j+1}, \ldots, X_{p}\right)
\end{aligned}
$$

pour tout $j \in\{1, \ldots, p\}$. On suppose que $P$ est de degré au plus $A(n+1)-2$ par rapport à chacune des variables. Alors la série

$$
\sum_{k_{1} \geq \cdots \geq k_{p} \geq 1} \frac{P\left(k_{1}, \ldots, k_{p}\right)}{\left(k_{1}\right)_{n+1}^{A} \cdots\left(k_{p}\right)_{n+1}^{A}}
$$

est convergente et c'est un polynôme à coefficients rationnels en les quantités

$$
\sum_{\sigma \in \mathfrak{S}_{q}} \varepsilon(\sigma) \zeta\left(s_{\sigma(1)}, \ldots, s_{\sigma(q)}\right)
$$

avec $q \in\{1, \ldots, p\}$ et $s_{1}, \ldots, s_{q} \geq 3$ impairs.

La somme (1.5) est appelée polyzêta antisymétrique dans [12]. Lorsque $q=1$, il s'agit simplement de $\zeta\left(s_{1}\right)$; pour $q=2$, on obtient $\zeta\left(s_{1}, s_{2}\right)-\zeta\left(s_{2}, s_{1}\right)$. L'énoncé plus précis donné dans [12] montre notamment que lorsque $p=1$, on obtient une forme linéaire en 1 et les $\zeta(s)$, pour $s$ impair compris entre 3 et $A$. Quand $p=2$, on obtient une forme linéaire en 1 , les $\zeta(s)$ pour $s$ impair compris entre 3 et $2 A$, et les $\zeta\left(s, s^{\prime}\right)-\zeta\left(s^{\prime}, s\right)$ pour $s, s^{\prime}$ impairs tels que $3 \leq s<s^{\prime} \leq$ $A^{(4)}$.

Dans le même esprit que le théorème 1.6, les tests effectués grâce à l'algorithme [11] ont permis de deviner puis de démontrer d'autres propriétés de symétrie [15].

Enfin, une dernière difficulté, et non la moindre, consiste à obtenir des résultats diophantiens en direction des conjectures 1.1 et 1.2 à l'aide de l'approche combinatoire développée ici. Nous nous contentons ici de démontrer un théorème « technique » concernant le dénominateur commun aux coefficients rationnels des combinaisons linéaires produites par certaines séries du type de (1.2) : voir le théorème 5.1 au paragraphe 5 .

Remerciements. - Nous avons pu faire fonctionner notre implémentation de l'algorithme présenté dans ce texte sur la grappe Médicis. Cela nous a permis de diminuer les temps de calcul nécessaires. En outre, le premier auteur remercie l'IHES pour son hospitalité durant la rédaction de ce travail.

(4) Les trois premiers exemples numériques précédant le théorème 1.6 suggèrent que, pour $p=2$, on a parfois des zêtas simples jusqu'à $2 A-3$ et des zêtas doubles avec $3 \leq s<s^{\prime} \leq A-2$ seulement. Nous n'avons pas cherché à savoir sous quelles conditions cela est vrai. 


\section{Liens avec les intégrales hypergéométriques}

Dans ce paragraphe, on s'intéresse au lien entre certaines intégrales multiples naturellement liées aux polyzêtas et les séries multiples que nous considérons dans le présent article. À nos yeux, la souplesse combinatoire des séries semble bien adaptée à la construction de formes linéaires en polyzêtas mais l'utilisation d'une intégrale ou d'une série dans ce but est essentiellement une affaire de goût, chacune ayant des avantages et des inconvénients. De plus, nous mentionnons certaines intégrales dont on sait qu'elles s'expriment à l'aide de polyzêtas mais auxquelles nos méthodes ne s'appliquent pas.

2.1. Exemples. - Il n'est pas possible de citer l'ensemble des intégrales multiples hypergéométriques qui sont apparues dans la littérature et nous ne mentionnons que les exemples les plus connus.

Posons, pour tous entiers $A \geq 2$ et $n \geq 0$,

$$
J_{A, n}=\int_{[0,1]^{A}} \frac{\prod_{j=1}^{A} x_{j}^{n}\left(1-x_{j}\right)^{n}}{Q_{A}\left(x_{1}, x_{2}, \ldots, x_{A}\right)^{n+1}} \mathrm{~d} x_{1} \cdots \mathrm{d} x_{A},
$$

où $Q_{A}(\underline{x})=1-\left(\cdots\left(1-\left(1-x_{A}\right) x_{A-1}\right) \cdots\right) x_{1}$. Lorsque $A=2$ ou $A=3$, on retrouve les célèbres intégrales de Beukers [6], qui a redémontré le théorème d'Apéry en utilisant le fait que $J_{2, n} \in \mathbb{Q}+\mathbb{Q} \zeta(2)$ et $J_{3, n} \in \mathbb{Q}+\mathbb{Q} \zeta(3)$, sous une forme plus précise. En restant en dimension $A=2$ ou $A=3$, ces intégrales ont ensuite été généralisées dans le but d'améliorer les mesures d'irrationalité respectives de $\zeta(2)$ et $\zeta(3)$ : le point d'orgue est la «méthode du groupe » de Rhin-Viola [31], [32], qui ont suivi des travaux de Hata [21], [22] en particulier. La principale difficulté de cette approche consiste à montrer directement que ces intégrales sont bien des formes linéaires en les valeurs de zêta.

En dimension supérieure, Vasilyev [41] a formulé la conjecture suivante, qu'il a prouvée pour $A=4$ et 5 :

Pour tous entiers $A \geq 2$ et $n \geq 0$, il existe des rationnels $\left(p_{j, A, n}\right)_{j=0,2,3, \ldots, A}$ tels que

$$
J_{A, n}=p_{0, A, n}+\sum_{\substack{2 \leq j \leq A \\ j \equiv A(\bmod 2)}} p_{j, A, n} \zeta(j)
$$

Cette conjecture, dont l'attaque directe est très difficile, a été démontrée par Zudilin $[48, \S 8]$ au moyen d'une identité inattendue entre les intégrales $J_{A, n}$ et certaines séries hypergéométriques très bien équilibrées. Comme on le montre au paragraphe 3.1, il est alors assez facile d'obtenir une forme linéaire en valeurs de zêta à partir d'une série hypergéométrique simple.

TOME $136-2008-\mathrm{N}^{\mathrm{O}} 1$ 
Il existe par ailleurs des intégrales d'une forme assez différente et qui ont été étudiées principalement par Sorokin [35], [36]. Dans [36], il a obtenu une preuve alternative du théorème d'Apéry en montrant que

$$
S_{3, n}=\int_{[0,1]^{3}} \frac{x^{n}(1-x)^{n} y^{n}(1-y)^{n} z^{n}(1-z)^{n}}{(1-x y)^{n+1}(1-x y z)^{n+1}} \mathrm{~d} x \mathrm{~d} y \mathrm{~d} z \in \mathbb{Q}+\mathbb{Q} \zeta(3),
$$

tandis que dans [35], il a obtenu une nouvelle preuve de la transcendance de $\pi$ en utilisant l'intégrale

$$
T_{A, n}=\int_{[0,1]^{2 A}} \prod_{j=1}^{A} \frac{\left(x_{j} y_{j}\right)^{n+(A-j)(n+1)}\left(1-x_{j}\right)^{n}\left(1-y_{j}\right)^{n}}{\left(1-x_{1} y_{1} \cdots x_{j} y_{j}\right)^{n+1}} \mathrm{~d} x_{j} \mathrm{~d} y_{j},
$$

dont il a montré qu'elle était une forme linéaire rationnelle en 1 et les $\zeta(2,2, \ldots, 2)=\pi^{2 j} /(2 j+1)$ !, pour $j=1, \ldots, A$. Dans les deux cas, Sorokin parvient à exprimer ses intégrales comme combinaison linéaire de valeurs de polyzêtas en résolvant de manière itérative des problèmes de Padé non triviaux. D'une manière générale, lorsqu'une intégrale provient d'un problème de Padé explicite, il arrive que l'énoncé même du problème permette d'éliminer a priori certains polyzêtas des formes linéaires lorsque l'on spécialise les polylogarithmes multiples en 1 ou en une autre valeur intéressante. Ceci confère un grand avantage à cette approche lorsqu'on peut la mettre en œuvre mais elle semble difficile à généraliser. De fait, les travaux ultérieurs cherchent tous à s'affranchir de l'étape « Padé».

Le fait particulièrement remarquable que $J_{3, n}=S_{3, n}$ pour tout entier $n \geq 0$ a été généralisé par Fischler [16] et Zlobin [45] indépendamment, qui ont montré entre autres choses que l'on a les identités

$$
J_{A, n}=\int_{[0,1]^{A}} \prod_{j=1}^{\frac{1}{2} A} \frac{x_{j}^{n}\left(1-x_{j}\right)^{n} y_{j}^{n}\left(1-y_{j}\right)^{n}}{\left(1-x_{1} y_{1} \cdots x_{j} y_{j}\right)^{n+1}} \mathrm{~d} x_{j} \mathrm{~d} y_{j}
$$

pour $A \geq 2$ pair et

$$
\begin{aligned}
J_{A, n}=\int_{[0,1]^{A}} \frac{z^{n}(1-z)^{n}}{\left(1-x_{1} y_{1} \cdots x_{a} y_{a} z\right)^{n+1}} & \\
& \left(\prod_{j=1}^{a} \frac{x_{j}^{n}\left(1-x_{j}\right)^{n} y_{j}^{n}\left(1-y_{j}\right)^{n}}{\left(1-x_{1} y_{1} \cdots x_{j} y_{j}\right)^{n+1}} \mathrm{~d} x_{j} \mathrm{~d} y_{j}\right) \mathrm{d} z
\end{aligned}
$$

pour $A \geq 3$ impair avec $a=\frac{1}{2}(A-1)$. Il découle de ces travaux l'intuition assez nette que l'on ne perd rien à travailler avec des généralisations de l'une ou l'autre des intégrales $J_{A, n}$ et $S_{A, n}$. Il s'avère que les intégrales de Sorokin $S_{A, n}$ à droite de (2.3) et (2.4) se développent un peu plus facilement en séries multiples que les intégrales $J_{A, n}$ et qu'elles donnent immédiatement des polyzêtas dans le cas $n=0$. Dans une perspective diophantienne, il est donc 
naturel de produire des formes linéaires en polyzêtas à partir d'intégrales du type de Sorokin les plus générales possibles; une telle relation a été démontrée par Zlobin [45]. La proposition 2.1 (démontrée au paragraphe 2.2 ci-dessous) couplée aux résultats de cet article nous permet de redémontrer une assertion similaire à celle de Zlobin mais nous insistons ici sur le fait que nos résultats (résumés informellement par le théorème 1.3) nous permettent de traiter des séries multiples plus générales que celles apparaissant dans la proposition 2.1 ou dans les travaux de Zlobin.

Terminons ce paragraphe en mentionnant un récent article de Zlobin [47], où il obtient une nouvelle preuve de la conjecture de Vasilyev en partant de l'intégrale $S_{A, n}$ convenablement développée en série multiple : il s'agit d'un remarquable tour de force.

2.2. Développement en série de certaines intégrales de Sorokin. - Le but de ce paragraphe est d'exprimer une intégrale de type Sorokin générale (elle contient du moins tous les cas mentionnés ci-dessus) comme une série multiple. Cette dernière est un cas particulier de celle que nous développons en polylogarithmes multiples et/ou polyzêtas dans la suite de l'article : pour ceux qui aiment travailler à partir d'intégrales, la proposition 2.1 (voir aussi le lemme 2 de [46]) est donc la première étape de notre algorithme de construction de formes linéaires en polyzêtas.

Proposition 2.1. - Soient des entiers $D, p \geq 1$ et des entiers positifs $r_{1}, \ldots, r_{p}, s_{1}, \ldots, s_{p}, t_{1}, \ldots, t_{p}$ et $0=d_{0}<d_{1}<d_{2}<\cdots<d_{p}=D$. Pour tout nombre complexe $z$ tel que $|z|>1$, on a l'identité

$$
\begin{aligned}
\int_{[0,1]^{D}} & \prod_{j=1}^{p} \frac{\prod_{\ell=d_{j-1}+1}^{d_{j}} x_{\ell}^{r_{j}}\left(1-x_{\ell}\right)^{s_{j}}}{\left(z-x_{1} \cdots x_{d_{j}}\right)^{t_{j}+1}} \mathrm{~d} x_{j} \\
= & z^{-\left(t_{1}+\cdots+t_{p}+p-1\right)} \prod_{j=1}^{p} \frac{s_{j} !^{A_{j}}}{t_{j} !} \times \sum_{k_{1} \geq \cdots \geq k_{p} \geq 1} z^{-k_{1}} \prod_{j=1}^{p} \frac{\left(k_{j}-k_{j+1}+1\right)_{t_{j}}}{\left(k_{j}+r_{j}\right)_{s_{j}+1}^{A_{j}}}
\end{aligned}
$$

où $k_{p+1}=1$ et $A_{j}=d_{j}-d_{j-1}$ pour $j=1, \ldots, p$. La série est de profondeur $p$ et de poids $D$.

Remarque 2. - L'équation (2.5) s'étend à $|z|=1$ lorsque les deux membres ont un sens simultanément.

Dans les applications diophantiennes, il est pratique de sommer sur des indices $K_{j}$ définis par $K_{j}=k_{j}+r$, où $r=\min r_{j}$. En particulier, si tous les $r_{j}$ sont égaux à $r$, la série s'écrit

$$
\sum_{K_{1} \geq \cdots \geq K_{p} \geq r+1} z^{-K_{1}+r} \prod_{j=1}^{p} \frac{\left(K_{j}-K_{j+1}+1\right)_{t_{j}}}{\left(K_{j}\right)_{s_{j}+1}^{A_{j}}}
$$

TOME $136-2008-\mathrm{N}^{\mathrm{O}} 1$ 
avec $K_{p+1}=r+1$. De plus, si $t_{p}=r$, la présence du symbole de Pochhammer $\left(K_{p}-r\right)_{r}$ implique que sommer sur l'ensemble d'indices $K_{1} \geq \cdots \geq K_{p} \geq r+1$ revient au même que sommer sur $K_{1} \geq \cdots \geq K_{p} \geq 1$.

Démonstration. - Supposer que $|z|>1$ assure que les diverses décompositions en séries et inversions séries-intégrales ci-dessous sont licites. Le cas d'un point du cercle $|z|=1$ s'obtient en invoquant des critères de continuité (théorèmes d'Abel, de Lebesgue, etc.).

On développe le dénominateur de l'intégrale multiple, notée $I(z)$ dans la suite, au moyen de l'identité (avec $|z|>1,0 \leq x \leq 1$ ) :

$$
\frac{1}{(z-x)^{t+1}}=\frac{1}{z^{t}} \sum_{m=0}^{\infty}\left(\begin{array}{c}
m+t \\
m
\end{array}\right)\left(\frac{x}{z}\right)^{m}
$$

et on obtient alors

$$
\begin{aligned}
& I(z)=z^{-\left(t_{1}+\cdots+t_{p}+p\right)} \sum_{m_{1}, \ldots, m_{p} \geq 0} \prod_{j=1}^{p}\left(\begin{array}{c}
m_{j}+t_{j} \\
m_{j}
\end{array}\right) z^{-m_{j}} \\
& \times \int_{[0,1]^{D}} \prod_{j=1}^{p}\left(\left(x_{1} \cdots x_{d_{j}}\right)^{m_{j}} \prod_{\ell=d_{j-1}+1}^{d_{j}} x_{\ell}^{r_{j}}\left(1-x_{\ell}\right)^{s_{j}} \mathrm{~d} x_{j}\right) .
\end{aligned}
$$

Or on vérifie que

$$
\prod_{j=1}^{p}\left(\left(x_{1} \cdots x_{d_{j}}\right)^{m_{j}} \prod_{\ell=d_{j-1}+1}^{d_{j}} x_{\ell}^{r_{j}}\left(1-x_{\ell}\right)^{s_{j}}\right)=\prod_{j=1}^{p}\left(\prod_{\ell=d_{j-1}+1}^{d_{j}} x_{\ell}^{r_{j}+m_{j}+\cdots+m_{p}}\left(1-x_{\ell}\right)^{s_{j}}\right) .
$$

On peut séparer les variables dans l'intégrale et on obtient alors $D$ intégrales facilement calculables (ce sont des fonctions Beta d'Euler), d'où

$$
\begin{aligned}
I(z) & =z^{-\left(t_{1}+\cdots+t_{p}+p\right)} \sum_{m_{1}, \ldots, m_{p} \geq 0} \\
& \times \prod_{j=1}^{p} \frac{z^{-m_{j}}\left(\begin{array}{c}
m_{j}+t_{j} \\
m_{j}
\end{array}\right)}{\left(\begin{array}{c}
r_{j}+s_{j}+m_{j}+\cdots+m_{p} \\
s_{j}
\end{array}\right)^{A_{j}}\left(r_{j}+s_{j}+m_{j}+\cdots+m_{p}+1\right)^{A_{j}}} .
\end{aligned}
$$

On utilise maintenant les transformations triviales

$$
\begin{aligned}
&\left(\begin{array}{c}
m_{j}+t_{j} \\
m_{j}
\end{array}\right)=\frac{\left(m_{j}+1\right)_{t_{j}}}{t_{j} !}, \\
&\left(\begin{array}{c}
r_{j}+s_{j}+m_{j}+\cdots+m_{p} \\
s_{j}
\end{array}\right)\left(r_{j}+s_{j}+m_{j}+\cdots+m_{p}+1\right) \\
&=\frac{\left(r_{j}+m_{j}+\cdots+m_{p}+1\right)_{s_{j}+1}}{s_{j} !}
\end{aligned}
$$


et on pose $k_{j}=m_{j}+\cdots+m_{p}+1$ pour $j=1, \ldots, p$, ainsi que $k_{p+1}=1$. On obtient alors

$$
I(z)=z^{-\left(t_{1}+\cdots+t_{p}+p-1\right)} \prod_{j=1}^{p} \frac{s_{j} !^{A_{j}}}{t_{j} !} \times \sum_{k_{1} \geq \cdots \geq k_{p} \geq 1} z^{-k_{1}} \prod_{j=1}^{p} \frac{\left(k_{j}-k_{j+1}+1\right)_{t_{j}}}{\left(k_{j}+r_{j}\right)_{s_{j}+1}^{A_{j}}},
$$

ce qui termine la preuve.

À titre d'exemples, remarquons que l'intégrale $S_{3, n}$ en (2.1) vaut exactement la série (1.3) donnée dans l'introduction tandis que l'intégrale (2.2) s'exprime de la manière suivante, en posant $k_{A+1}=n+1$ :

$$
T_{A, n}=n !^{A} \sum_{k_{1} \geq \cdots \geq k_{A} \geq 1} \prod_{j=1}^{A} \frac{\left(k_{j}-k_{j+1}+1\right)_{n}}{\left(k_{j}+(A-j)(n+1)\right)_{n+1}^{2}} .
$$

Notre algorithme permet ensuite d'exprimer effectivement ces intégrales comme des formes linéaires en polyzêtas. Jusqu'en poids 4 , on ne voit apparaître que des valeurs de zêta car tous les polyzêtas de poids $\leq 4$ sont des multiples rationnels de $1, \zeta(2), \zeta(3)$ ou $\zeta(4)$. En revanche, à partir du poids 5 , on doit s'attendre à obtenir des polyzêtas linéairement indépendants (du moins, conjecturalement) des valeurs de zêta, comme le montre l'exemple de l'intégrale

$$
\int_{[0,1]^{5}} \frac{\prod_{j=1}^{5} x_{j}^{n}\left(1-x_{j}\right)^{n} \mathrm{~d} x_{j}}{\left(1-x_{1} x_{2} x_{3}\right)^{n+1}\left(1-x_{1} x_{2} x_{3} x_{4} x_{5}\right)^{n+1}} .
$$

Pour $n=0$, elle vaut $\zeta(3,2)=-\frac{11}{2} \zeta(5)+3 \zeta(2) \zeta(3)$, qui n'est donc probablement pas un multiple rationnel d'une valeur de zêta en un entier. Pour $n=1,2$ et 3 , elle est une combinaison linéaire rationnelle en $\zeta(2), \zeta(3), \zeta(4), \zeta(5), \zeta(2,2)$ et $\zeta(3,2)$. Le coefficient de $\zeta(3,2)$ dans ces combinaisons linéaires est non nul; on peut l'expliciter comme une somme double finie, ce qui pourrait peut-être permettre de démontrer qu'il est non nul pour tout $n \geq 4$, si nécessaire.

2.3. D'autres exemples d'intégrales hypergéométriques. - Il existe beaucoup d'intégrales hypergéométriques (hormis celles de Vasilyev et Sorokin) dont on sait démontrer, par des moyens plus ou moins détournés, qu'elles s'expriment comme formes linéaires en polyzêtas. Les deux exemples que nous allons aborder sont dus à Zudilin et Goncharov-Manin respectivement.

L'intégrale considérée par Zudilin [50] est la suivante :

$$
Z_{n}=\int_{[0,1]^{5}} \frac{\prod_{j=1}^{5} x_{j}^{n}\left(1-x_{j}\right)^{n} \mathrm{~d} x_{j}}{Q\left(x_{1}, x_{2}, x_{3}, x_{4}, x_{5}\right)^{n+1}}
$$

où $Q(\underline{x})=x_{1}\left(1-\left(1-\left(1-\left(1-x_{2}\right) x_{3}\right) x_{4}\right) x_{5}\right)+\left(1-x_{1} x_{2} x_{3} x_{4} x_{5}\right)$. Par un procédé indirect (basé sur des transformations hypergéométriques), il montre que $Z_{n}$ 
est égale à une série de nature hypergéométrique très bien équilibrée (avec une dérivation double du sommande) et il en déduit que $Z_{n} \in \mathbb{Q}+\mathbb{Q} \zeta(4)$.

Les intégrales de Goncharov-Manin [20] apparaissent quant à elles comme des périodes de certains motifs de Tate mixtes, dont Brown [7] a donné la forme explicite suivante :

$$
\int_{[0,1]^{A}} \frac{\prod_{j=1}^{A} x_{j}^{r_{j}}\left(1-x_{j}\right)^{s_{j}} \mathrm{~d} x_{j}}{\prod_{1 \leq i<j \leq A}\left(1-x_{i} \cdots x_{j}\right)^{t_{i, j}}}
$$

avec des entiers $r_{j}, s_{j}, t_{i, j} \geq 0$ tels que l'intégrale converge. Remarquons que (2.6) contient comme cas particulier les intégrales abordées par la proposition 2.1 (en $z=1$ ). Par des arguments de nature géométrique, Brown a prouvé une conjecture de Goncharov-Manin qui affirmait que ces intégrales sont toujours des formes linéaires rationnelles en polyzêtas. La construction qu'il fournit est théoriquement effective, mais en pratique elle ne permet pas de décomposer facilement une telle intégrale. Ceci rend impossible une quelconque utilisation diophantienne de son théorème par les voies classiques.

Ces deux types d'intégrales ont donc le défaut de n'être évaluables que par des procédés très indirects. Pour remédier à cela, on pourrait tenter de les « développer » en séries multiples à la manière de la proposition 2.1, puis espérer appliquer une généralisation convenable de notre algorithme. Ceci n'aura rien d'évident; par exemple, les cas les plus simples de l'intégrale (2.6) peuvent conduire à des séries telles que

$$
\sum_{m, n \geq 1} \frac{1}{m^{s_{1}} n^{s_{2}}(m+n)^{s_{3}}},
$$

dont il n'est même pas clair a priori qu'elles puissent s'exprimer à l'aide de polyzêtas (c'est cependant bien le cas : voir par exemple [14] pour plus de détails et des références). Étendre notre algorithme nécessitera donc des idées nouvelles.

\section{3. Étude de deux situations instructives}

3.1. Le cas de la profondeur 1. - La stratégie ${ }^{(5)}$ pour démontrer les théorèmes diophantiens concernant les valeurs de la fonction zêta est la suivante. Soient

(5) C'est essentiellement la seule dont on dispose : toutes les autres approches connues produisent les mêmes formes linéaires (voir [17]). 
des entiers $n \geq 0, A \geq 1$ et $P(X) \in \mathbb{Q}[X]$. Considérons la fraction rationnelle $R(X)=P(X) /(X)_{n+1}^{A}$ ainsi que la série

$$
S(z)=\sum_{k=1}^{\infty} R(k) z^{-k} .
$$

On suppose que cette dernière est convergente pour $z=1$, ce qui signifie $\operatorname{deg}(P) \leq A(n+1)-2$. On commence par développer $R(X)$ en éléments simples :

$$
R(X)=\sum_{s=1}^{A} \sum_{j=0}^{n} \frac{C\left[\begin{array}{l}
s \\
j
\end{array}\right]}{(X+j)^{s}}
$$

avec

$$
C\left[\begin{array}{l}
s \\
j
\end{array}\right]=\left.\frac{1}{(A-s) !}\left(R(X)(X+j)^{A}\right)^{(A-s)}\right|_{X=-j}
$$

et, en reportant dans $S(z)$, on obtient lorsque $|z|>1$

$$
S(z)=\sum_{s=1}^{A} \sum_{j=0}^{n} C\left[\begin{array}{l}
s \\
j
\end{array}\right] \sum_{k=1}^{\infty} \frac{z^{-k}}{(k+j)^{s}} .
$$

On remarque alors que, trivialement,

$$
\sum_{k=1}^{\infty} \frac{z^{-k}}{(k+j)^{s}}=z^{j} \operatorname{Li}_{s}\left(\frac{1}{z}\right)-\sum_{k=1}^{j} \frac{z^{j-k}}{k^{s}}
$$

et donc qu'il existe des polynômes $Q_{s}(z) \in \mathbb{Q}[z]$, de degré au plus $n$, tels que

$$
S(z)=Q_{0}(z)+\sum_{s=1}^{A} Q_{s}(z) \operatorname{Li}_{s}\left(\frac{1}{z}\right) .
$$

On a bien sûr $\operatorname{Li}_{s}(1)=\zeta(s)$ et $\operatorname{Li}_{s}(-1)=\left(2^{1-s}-1\right) \zeta(s)$ pour tout $s>1$. Pour $s \geq 1$, on a l'expression très simple

$$
Q_{s}(z)=\sum_{j=0}^{n} C\left[\begin{array}{l}
s \\
j
\end{array}\right] z^{j} .
$$

Pour les applications envisagées, il est important de se ramener à des coefficients entiers et on montre que $Q_{1}(1)=0$ et $\mathrm{d}_{n}^{A-s} Q_{s}(z) \in \mathbb{Z}[z]$ pour tout $s \in\{0, \ldots, A\}$, où $\mathrm{d}_{n}=\operatorname{ppcm}\{1,2, \ldots, n\}$. Il existe donc des entiers $q_{s}$ tels que

$$
\mathrm{d}_{n}^{A} S(1)=q_{0}+\sum_{s=2}^{A} q_{s} \zeta(s),
$$

et une expression similaire pour $S(-1)$. 
Tout le problème réside maintenant dans des choix de $A$ et de $P$ tels que l'on puisse appliquer efficacement un critère d'irrationalité ou d'indépendance linéaire : il apparaît rapidement que l'on doit éliminer les nombres $\zeta(s)$ pour $s$ pair, sous peine de n'obtenir que des résultats triviaux. Une manière d'y parvenir est d'imposer que le polynôme $P(X)$ satisfasse à

$$
P(-X-n)=-P(X) \text {. }
$$

En effet, par unicité de la décomposition de $R(X)$ en éléments simples, l'équation (3.2) se traduit par $C\left[\begin{array}{c}s \\ n-j\end{array}\right]=(-1)^{A(n+1)+s+1} C\left[\begin{array}{l}s \\ j\end{array}\right]$ et donc les coefficients $q_{s}$ sont nuls pour $s$ pair lorsque $A$ est lui-même pair. Par exemple, lorsque $A$ est pair, on peut utiliser les séries

$$
n !^{A-2 r} \sum_{k=1}^{\infty}\left(k+\frac{n}{2}\right) \frac{(k-r n)_{r n}(k+n+1)_{r n}}{(k)_{n+1}^{A}}=q_{0}+\sum_{\substack{s=3 \\ s \text { impair }}}^{A} q_{s} \zeta(s),
$$

qui sont des séries hypergéométriques particulières, dites very-well-poised (voir [4], [34] pour la définition exacte). On se réfèrera à [5], [17], [23], [33], [49] pour plus de détails sur l'utilisation diophantienne de ce type de série.

3.2. Le cas de la profondeur 2. - Une fois formalisé le cas de la profondeur 1 , il est naturel d'essayer de suivre la même démarche en profondeur supérieure. Le cas de la profondeur $p=2$ est déjà instructif et nous allons le traiter en détails.

Nous expliquons notre approche sur la série suivante

$$
S\left(z_{1}, z_{2}\right)=\sum_{k_{1} \geq k_{2} \geq 1} \frac{P\left(k_{1}, k_{2}\right)}{\left(k_{1}\right)_{n+1}^{2}\left(k_{2}\right)_{n+1}^{2}} z_{1}^{-k_{1}} z_{2}^{-k_{2}},
$$

avec $\operatorname{deg}_{k_{1}}(P) \leq 2(n+1)-2$ et $\operatorname{deg}_{k_{2}}(P) \leq 2(n+1)-2$, ce qui implique que la série converge absolument pour $\left|z_{1}\right| \geq 1$ et $\left|z_{2}\right| \geq 1$. On notera que cette condition suffisante de convergence n'est pas une condition nécessaire; par exemple la série introduite en (1.3) ne la vérifie pas. Les conséquences de cela sont évoquées à la fin de ce paragraphe.

La première étape consiste, comme précédemment, à décomposer en éléments simples la fraction rationnelle qui constitue le sommande de $S\left(z_{1}, z_{2}\right)$ :

$$
\frac{P\left(k_{1}, k_{2}\right)}{\left(k_{1}\right)_{n+1}^{2}\left(k_{2}\right)_{n+1}^{2}}=\sum_{j_{1}, j_{2}=0}^{n} \sum_{s_{1}, s_{2}=1}^{2} \frac{C\left[\begin{array}{l}
s_{1}, s_{2} \\
j_{1}, j_{2}
\end{array}\right]}{\left(k_{1}+j_{1}\right)^{s_{1}}\left(k_{2}+j_{2}\right)^{s_{2}}},
$$

où les $C\left[\begin{array}{l}s_{1}, s_{2} \\ j_{1}, j_{2}\end{array}\right]$ sont des rationnels explicitables. Il est important de noter que la condition portant sur les degrés de $P$ implique que cette décomposition n'a 
pas de partie entière. En reportant dans $S\left(z_{1}, z_{2}\right)$, on obtient ainsi

$$
S\left(z_{1}, z_{2}\right)=\sum_{j_{1}, j_{2}=0}^{n} \sum_{s_{1}, s_{2}=1}^{2} C\left[\begin{array}{l}
s_{1}, s_{2} \\
j_{1}, j_{2}
\end{array}\right] \sum_{k_{1} \geq k_{2} \geq 1} \frac{z_{1}^{-k_{1}} z_{2}^{-k_{2}}}{\left(k_{1}+j_{1}\right)^{s_{1}}\left(k_{2}+j_{2}\right)^{s_{2}}} .
$$

La deuxième étape consiste à exprimer explicitement la série

$$
\sum_{k_{1}=1}^{\infty} \frac{z_{1}^{-k_{1}}}{\left(k_{1}+j_{1}\right)^{s_{1}}} \sum_{k_{2}=1}^{k_{1}} \frac{z_{2}^{-k_{2}}}{\left(k_{2}+j_{2}\right)^{s_{2}}}
$$

comme une combinaison linéaire à coefficients dans $\mathbb{Q}\left[z_{1}^{ \pm 1}, z_{2}^{ \pm 1}\right]$ en les polylogarithmes multiples (larges ou stricts). Comme on l'a vu en (3.1), dans le cas d'une seule variable ( $p=1)$, c'est une étape triviale mais, malheureusement, en deux variables, ce n'est plus le cas. On écrit tout d'abord la somme intérieure sur $k_{2}$ comme

$$
\begin{aligned}
\sum_{k_{2}=1}^{k_{1}} \frac{z_{2}^{-k_{2}}}{\left(k_{2}+j_{2}\right)^{s_{2}}} & =\sum_{k_{2}=j_{2}+1}^{k_{1}+j_{2}} \frac{z_{2}^{j_{2}-k_{2}}}{k_{2}^{s_{2}}} \\
& =\left(\sum_{k_{2}=1}^{k_{1}+j_{1}}-\sum_{k_{2}=1}^{j_{2}}+\varepsilon_{j_{1}, j_{2}} \sum_{k_{2}=k_{1}+j_{1} \wedge j_{2}+1}^{k_{1}+j_{1} \vee j_{2}}\right) \frac{z_{2}^{j_{2}-k_{2}}}{k_{2}^{s_{2}}}
\end{aligned}
$$

où $j_{1} \wedge j_{2}=\min \left(j_{1}, j_{2}\right), j_{1} \vee j_{2}=\max \left(j_{1}, j_{2}\right)$ et

$$
\varepsilon_{j_{1}, j_{2}}=\left\{\begin{aligned}
1 & \text { si } j_{1}<j_{2}, \\
-1 & \text { si } j_{1}>j_{2}, \\
0 & \text { si } j_{1}=j_{2} .
\end{aligned}\right.
$$

Puis on reporte ces trois sommes dans la somme sur $k_{1}$. Les deux premières séries se traitent facilement :

$$
\begin{aligned}
\sum_{k_{1}=1}^{\infty} \frac{z_{1}^{-k_{1}}}{\left(k_{1}+j_{1}\right)^{s_{1}}} & \sum_{k_{2}=1}^{k_{1}+j_{1}} \frac{z_{2}^{j_{2}-k_{2}}}{k_{2}^{s_{2}}}=\sum_{k_{1}=j_{1}+1}^{\infty} \frac{z_{1}^{j_{1}-k_{1}}}{k_{1}^{s_{1}}} \sum_{k_{2}=1}^{k_{1}} \frac{z_{2}^{j_{2}-k_{2}}}{k_{2}^{s_{2}}} \\
& =z_{1}^{j_{1}} z_{2}^{j_{2}} \mathrm{La}_{s_{1}, s_{2}}\left(\frac{1}{z_{1}}, \frac{1}{z_{2}}\right)-\sum_{k_{1}=1}^{j_{1}} \frac{z_{1}^{j_{1}-k_{1}}}{k_{1}^{s_{1}}} \sum_{k_{2}=1}^{k_{1}} \frac{z_{2}^{j_{2}-k_{2}}}{k_{2}^{s_{2}}}, \\
\sum_{k_{1}=1}^{\infty} \frac{z_{1}^{-k_{1}}}{\left(k_{1}+j_{1}\right)^{s_{1}}} \sum_{k_{2}=1}^{j_{2}} \frac{z_{2}^{j_{2}-k_{2}}}{k_{2}^{s_{2}}} & =z_{1}^{j_{1}}\left(\sum_{k_{2}=1}^{j_{2}} \frac{z_{2}^{j_{2}-k_{2}}}{k_{2}^{s_{2}}}\right) \operatorname{La}_{s_{1}}\left(\frac{1}{z_{1}}\right)-\left(\sum_{k_{1}=1}^{j_{1}} \frac{z_{1}^{j_{1}-k_{1}}}{k_{1}^{s_{1}}}\right)\left(\sum_{k_{2}=1}^{j_{2}} \frac{z_{2}^{j_{2}-k_{2}}}{k_{2}^{s_{2}}}\right) .
\end{aligned}
$$

TOME $136-2008-\mathrm{N}^{\mathrm{O}} 1$ 
La troisième série est un peu plus compliquée : on note que

$$
\begin{aligned}
\sum_{k_{1}=1}^{\infty} \frac{z_{1}^{-k_{1}}}{\left(k_{1}+j_{1}\right)^{s_{1}}} & \sum_{\substack{k_{2}=k_{1}+j_{1} \wedge j_{2}+1 \\
j_{1} \vee j_{2}}}^{k_{1}+j_{1} \vee j_{2}} \frac{z_{2}^{j_{2}-k_{2}}}{k_{2}^{s_{2}}} \\
= & \sum_{k_{2}=j_{1} \wedge j_{2}+1}^{j_{2}-k_{2}} \sum_{k_{1}=1}^{\infty} \frac{\left(z_{1} z_{2}\right)^{-k_{1}}}{\left(k_{1}+j_{1}\right)^{s_{1}}\left(k_{1}+k_{2}\right)^{s_{2}}}
\end{aligned}
$$

puis on développe en éléments simples (par rapport à la variable $k_{1}$ ) la fraction rationnelle

$$
\frac{1}{\left(k_{1}+j_{1}\right)^{s_{1}}\left(k_{1}+k_{2}\right)^{s_{2}}}
$$

pour conclure que cette série s'écrit comme une combinaison linéaire de $\mathrm{La}_{s}\left(1 / z_{1} z_{2}\right)$ avec $1 \leq s \leq s_{1} \vee s_{2}$ et aussi de $\operatorname{La}_{s_{1}+s_{2}}\left(1 / z_{1} z_{2}\right)$ si $k_{2}=j_{1} \vee j_{2}=j_{1}$, avec des coefficients polynomiaux en $z_{1}^{ \pm 1}$ et $z_{2}^{ \pm 1}$. En résumé, lorsque $z_{1}=1$ et $z_{2}=1$, la décomposition de la série (3.4) fait apparaître au plus les polyzêtas suivants : $\zeta\left(s_{1}, s_{2}\right), \zeta\left(s_{1}+s_{2}\right)$ et les $\zeta(s)$ pour $1 \leq s \leq s_{1} \vee s_{2}$. En particulier, il n'y a aucune raison apparente pour que les valeurs de zêta aux entiers pairs n'apparaissent pas.

Enfin, troisième étape, en reportant la décomposition ainsi obtenue dans (3.3), on doit identifier les polyzêtas qui apparaissent réellement dans $S(1,1)$, c'est-à-dire ceux affectés d'un coefficient non nul. Or cette identification n'est pas évidente : la série $S(1,1)$ fait apparaître a priori les polyzêtas dont certains sont divergents

$$
\zeta(1), \zeta(1,1), \zeta(2), \zeta(2,1), \zeta(1,2), \zeta(3), \zeta(2,2), \zeta(4) .
$$

Lorsque, par exemple, $P\left(X_{1}, X_{2}\right)=\left(X_{2}-n\right)_{n}\left(X_{1}-X_{2}+1\right)_{n}$, il est assez difficile de prouver ainsi que seuls $\zeta(2), \zeta(2,2)$ et $\zeta(4)$ n'ont pas un coefficient nul.

On doit aussi parfois tenir compte d'un autre phénomène : contrairement à $S\left(z_{1}, z_{2}\right)$, la décomposition en éléments simples du sommande de la série en (1.3) produit une partie entière (puisque le degré en $X_{2}$ de la fraction $\left(X_{2}-n\right)_{n}\left(X_{1}-X_{2}+1\right)_{n} /\left(X_{1}\right)_{n+1}^{2}\left(X_{2}\right)_{n+1}$ est positif) qui complique encore cette étape en faisant apparaître des polylogarithmes multiples « exotiques » tels que $\mathrm{La}_{2,-1}\left(z_{1}, z_{2}\right)$ qu'il faut traiter de façon ad hoc. Ce procédé devient quasiment inextricable en trois variables, ce qui explique le formalisme que nous développons au paragraphe 4 .

\section{Démonstration du théorème 1.3}

Nous venons de démontrer le théorème 1.3 pour $p=1$ (paragraphe 3.1 ) et, en partie, pour $p=2$ (paragraphe 3.2). Dans ce paragraphe, on le démontre en toute généralité : la stratégie consiste à se ramener dans un premier temps 
à un cas plus simple (paragraphe 4.1) que l'on démontre ensuite (théorème 4.1 au paragraphe 4.3). Nous en obtiendrons des raffinements au paragraphe 5 .

4.1. Décomposition des séries multiples en briques. - En imitant le cas de la profondeur 1, nous allons transformer la série

$$
\begin{gathered}
S_{P}\left[\begin{array}{c|c}
A_{1}, \ldots, A_{p} \\
n_{1}, \ldots, n_{p}
\end{array} \mid z_{1}, \ldots, z_{p}\right] \\
=\sum_{k_{1} \geq \cdots \geq k_{p} \geq 1} \frac{P\left(k_{1}, \ldots, k_{p}\right)}{\left(k_{1}\right)_{n_{1}+1}^{A_{1}} \cdots\left(k_{p}\right)_{n_{p}+1}^{A_{p}}} z_{1}^{-k_{1}} \cdots z_{p}^{-k_{p}}
\end{gathered}
$$

en développant en éléments simples la fraction rationnelle

$$
R\left(X_{1}, \ldots, X_{p}\right)=\frac{P\left(X_{1}, \ldots, X_{p}\right)}{\left(X_{1}\right)_{n_{1}+1}^{A_{1}} \cdots\left(X_{p}\right)_{n_{p}+1}^{A_{p}}} .
$$

Posons

$$
\widehat{A}_{i}=\operatorname{deg}_{X_{i}}(P)-A_{i}\left(n_{i}+1\right) .
$$

C'est le degré en $X_{i}$ de la fraction rationnelle $R$. Notons $J$ l'ensemble des indices $i \in\{1, \ldots, p\}$ tels que $\widehat{A}_{i} \geq 0$ (c'est-à-dire $\operatorname{deg}_{X_{i}}(P) \geq A_{i}\left(n_{i}+1\right)$ ) : c'est l'ensemble des $i$ pour lesquels $R$ est de degré positif ou nul en $X_{i}$, c'est-à-dire relativement auxquels une partie entière va apparaître. Pour $I \subset\{1, \ldots, p\}$, on note $I^{\mathrm{c}}=\{1, \ldots, p\} \backslash I$. Alors on a



$$
\begin{aligned}
& C\left[\begin{array}{c}
I \\
\left(s_{i}\right) \\
\left(j_{i}\right) \\
\left(\widehat{s}_{i}\right)
\end{array}\right] \frac{\prod_{i \in I} X_{i}^{\widehat{s}_{i}}}{\prod_{i \in I^{\mathrm{c}}}\left(X_{i}+j_{i}\right)^{s_{i}}}
\end{aligned}
$$

avec

$$
\begin{aligned}
& C\left[\begin{array}{c}
I \\
\left(s_{i}\right) \\
\left(j_{i}\right) \\
\left(\widehat{s}_{i}\right)
\end{array}\right]=\partial\left[\begin{array}{c}
I \\
\left(s_{i}\right) \\
\left(\widehat{s}_{i}\right)
\end{array}\right]\left(R_{I}\left(Y_{1}, \ldots, Y_{p}\right) \prod_{i \in I^{\mathrm{c}}}\left(Y_{i}+j_{i}\right)^{A_{i}} \prod_{i \in I} Y_{i}^{\widehat{A}_{i}}\right)_{\substack{Y_{i}=0 \text { pour } i \in I, Y_{i}=-j_{i} \text { pour } i \in I^{\mathrm{c}},}} \\
& \quad \text { томе } 136-2008-\mathrm{N}^{\mathrm{o}} 1
\end{aligned}
$$


en notant $\partial[\vdots]$ l'opérateur différentiel

$$
\partial\left[\begin{array}{c}
I \\
\left(s_{i}\right) \\
\left(\widehat{s}_{i}\right)
\end{array}\right]=\prod_{i \in I^{\mathrm{c}}}\left(\frac{1}{\left(A_{i}-s_{i}\right) !}\left(\frac{\partial}{\partial Y_{i}}\right)^{A_{i}-s_{i}}\right) \prod_{i \in I}\left(\frac{1}{\left(\widehat{A}_{i}-\widehat{s}_{i}\right) !}\left(\frac{\partial}{\partial Y_{i}}\right)^{\widehat{A}_{i}-\widehat{s}_{i}}\right)
$$

et $R_{I}\left(Y_{1}, \ldots, Y_{p}\right)$ la fraction rationnelle obtenue à partir de $R\left(X_{1}, \ldots, X_{p}\right)$ en posant

$$
X_{i}=\frac{1}{Y_{i}} \text { pour } i \in I \quad \text { et } \quad X_{i}=Y_{i} \text { pour } i \in I^{\mathrm{c}}
$$

Le cas particulier où il n'y a pas de partie entière correspond à $\widehat{A}_{i} \leq-1$ pour tout $i \in\{1, \ldots, p\}$, c'est-à-dire $J=\varnothing$. La somme sur $I$ se réduit alors à $I=\varnothing$, la famille $\left(\widehat{s}_{i}\right)$ est vide et on obtient la décomposition en éléments simples habituelle :

$R\left(X_{1}, \ldots, X_{p}\right)=\sum_{s_{1}=1}^{A_{1}} \cdots \sum_{s_{p}=1}^{A_{p}} \sum_{j_{1}=0}^{n_{1}} \cdots \sum_{j_{p}=0}^{n_{p}} C\left[\begin{array}{c}s_{1}, \ldots, s_{p} \\ j_{1}, \ldots, j_{p}\end{array}\right] \frac{1}{\left(X_{1}+j_{1}\right)^{s_{1}} \cdots\left(X_{p}+j_{p}\right)^{s_{p}}}$.

Revenons au cas général. En reportant (4.2) dans (4.1), on obtient

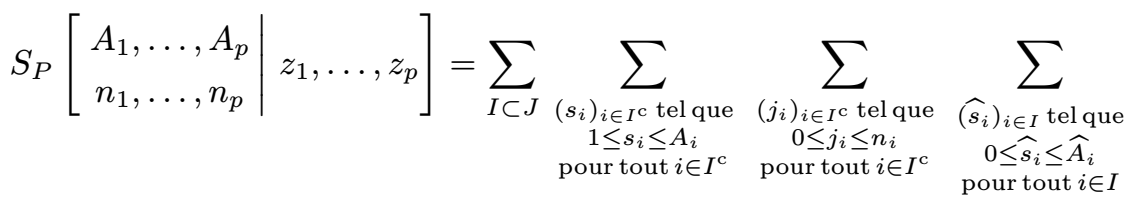

$$
\begin{aligned}
& C\left[\begin{array}{c}
I \\
\left(s_{i}\right) \\
\left(j_{i}\right) \\
\left(\widehat{s}_{i}\right)
\end{array}\right] \sum_{k_{1} \geq \cdots \geq k_{p} \geq 1} \frac{\prod_{i \in I} k_{i}^{\widehat{s_{i}}}}{\prod_{i \in I^{\mathrm{c}}}\left(k_{i}+j_{i}\right)^{s_{i}}} z_{1}^{-k_{1}} \cdots z_{p}^{-k_{p}} .
\end{aligned}
$$

On a donc ramené le problème initial (i.e., l'évaluation de (4.1)) à celui de la décomposition en polylogarithmes multiples de séries élémentaires de la forme

$$
\sum_{k_{1} \geq \cdots \geq k_{p} \geq 1} \frac{z_{1}^{-k_{1}} \cdots z_{p}^{-k_{p}}}{\left(k_{1}+j_{1}\right)^{s_{1}} \cdots\left(k_{p}+j_{p}\right)^{s_{p}}}
$$

où $s_{i} \in \mathbb{Z}$ et $j_{i} \in \mathbb{N}$. C'est ce problème que nous allons maintenant résoudre; cela terminera la preuve du théorème 1.3. 
4.2. Notations. - Dans tout ce paragraphe, $N$ désignera un entier $\geq 1$ qui jouera essentiellement le rôle de profondeur, rôle dévolu jusqu'à présent à l'entier $p$. On notera :

- $\underline{j}_{N}=\left(j_{i}\right)_{i=1, \ldots N}$ et $\underline{m}_{N}=\left(m_{i}\right)_{i=1, \ldots N}\left(\right.$ avec $\left.m_{1}=0\right)$ des suites de $\mathbb{N}$;

- $\underline{s}_{N}=\left(s_{i}\right)_{i=1, \ldots N}$ une suite d'entiers de $\mathbb{Z}$;

- $\underline{z}_{N}=\left(z_{i}\right)_{i=1, \ldots N}$ une suite de nombres complexes de modules $\geq 1$;

- $a \wedge b=\min (a, b)$ et $a \vee b=\max (a, b)$;

- $t_{p}=j_{p-1} \wedge\left(j_{p}+m_{p}\right)$ et $T_{p}=j_{p-1} \vee\left(j_{p}+m_{p}\right)($ pour $2 \leq p \leq N)$;

- $\varepsilon_{a, b}=1$ si $a<b,-1$ si $a>b, 0$ si $a=b$ et $\varepsilon_{p}=\varepsilon_{j_{p-1}, j_{p}+m_{p}}$ (pour $2 \leq p \leq N$ ).

À toute suite finie $\underline{u}_{N}=\left(u_{1}, \ldots, u_{N}\right)$, on associe les trois suites :

- $\underline{u}_{N}^{p}=\left(u_{p}, \ldots, u_{N}\right)$ de longueur $N-p+1($ pour $1 \leq p \leq N)$;

- ${ }_{p} \underline{u}_{N}=\left(u_{1}, \ldots, u_{p-2}, u_{p-1} u_{p}, u_{p+1}, \ldots, u_{N}\right)$ de longueur $N-1$ (pour $2 \leq p \leq N)$;

- $1 / \underline{u}_{N}=\left(1 / u_{1}, \ldots, 1 / u_{N}\right)$ lorsque les $u_{i}$ sont non nuls.

On définit les briques decalées-modulées par

$$
\begin{aligned}
& \mathrm{B}_{N}\left[\begin{array}{c|c}
\underline{s}_{N} & \\
\underline{m}_{N} & \underline{z}_{N} \\
\underline{j}_{N} &
\end{array}\right]=\sum_{k_{1} \geq 1} \frac{z_{1}^{-k_{1}} \cdots z_{N}^{-k_{N}}}{\left(k_{1}+j_{1}\right)^{s_{1}} \cdots\left(k_{N}+j_{N}\right)^{s_{N}}} . \\
& k_{1}+m_{2} \geq k_{2} \geq 1 \\
& \vdots \\
& k_{N-2}+m_{N-1} \geq k_{N-1} \geq 1 \\
& k_{N-1}+m_{N} \geq k_{N} \geq 1
\end{aligned}
$$

Les $j_{i}$ sont les décalages, les $m_{i}$ les modulations, les $s_{i}$ les exposants, $N$ la profondeur et on définit son poids comme étant $\sum_{p=1}^{N} \max \left(s_{p}, 0\right)$. Par définition, $m_{1}=0$ : toutes les briques $\mathrm{B}^{\prime}$ que nous construirons à l'aide de briques $\mathrm{B}$ avec $m_{1}=0$ auront aussi $m_{1}^{\prime}=0$. Ces séries convergent absolument lorsque $\left|z_{1}\right|>1$ et $\left|z_{j}\right| \geq 1$ pour $j=2, \ldots, N$, ce que l'on suppose dorénavant et qui légitime les diverses manipulations que nous effectuerons dessus; nous montrerons au paragraphe 7 comment obtenir des résultats similaires lorsque tous les $z_{i}$ valent 1 . Un cas particulier important est celui où tous les $m_{i}$ sont nuls : 
on parlera de brique décalée, ou simplement de brique ${ }^{(6)}$, et on la notera

$$
\mathrm{B}_{N}\left[\begin{array}{l|l}
\underline{s}_{N} & \underline{z}_{N} \\
\underline{j}_{N} & \underline{x}
\end{array}\right]=\sum_{k_{1} \geq \cdots \geq k_{N} \geq 1} \frac{z_{1}^{-k_{1}} \cdots z_{N}^{-k_{N}}}{\left(k_{1}+j_{1}\right)^{s_{1}} \cdots\left(k_{N}+j_{N}\right)^{s_{N}}} .
$$

Nous avons déjà rencontré ce type de briques dans les cas $N=1$ et $N=2$ au paragraphe 3 et en toute généralité au paragraphe 4.1. Pour obtenir des relations compactes, on définit la brique de profondeur 0 (et vide de paramètres) comme la fonction identiquement égale à 1 . La modulation semble a priori une notion artificielle et inutile puisqu'on ne s'intéresse réellement qu'aux briques décalées : à l'usage, il n'en est rien car, de façon surprenante, on ne peut apparemment pas produire le théorème 4.1 ci-dessous sans modulation.

Nous appellerons terme de profondeur $\leq N-1$ toute combinaison linéaire à coefficients dans $\mathbb{Q}\left[z_{1}^{ \pm 1}, \ldots, z_{N}^{ \pm 1}\right]$ de briques décalées-modulées de profondeur $\leq N-1$ et évaluées en des produits quelconques des variables $z_{1}, \ldots, z_{N}$. Le poids d'un terme de profondeur $N-1$ est le plus grand des poids des briques qui le composent.

Pour tout entier $p$ tel que $1 \leq p \leq N+1$, on définit le polynôme de Laurent

$$
Q_{\underline{s}_{N}^{p}, p}\left(K ; \underline{z}_{N}^{p}\right)=\sum_{K \geq k_{p} \geq \cdots \geq k_{N} \geq 1} \frac{z_{p}^{-k_{p}} \cdots z_{N}^{-k_{N}}}{\prod_{i=p}^{N} k_{i}^{s_{i}}}
$$

(qui vaut 0 si $K=0$ ) pour $p \leq N$ et $Q_{\underline{s}_{N}^{N+1}, N+1}\left(K ; \underline{z}_{N}^{N+1}\right)=1$ pour $p=N+1$. On notera

$$
Q_{\underline{s}_{N}^{p}, p}\left(K ; \underline{z}_{N}^{p}\right)=Q_{N, p}\left(K ; \underline{z}_{N}^{p}\right)
$$

lorsqu'il n'y aura pas de risque de confusion sur les exposants en jeu. On a

$$
Q_{N, p}\left(K ; \underline{z}_{N}^{p}\right)=\sum_{k_{p}=1}^{K} \frac{z_{p}^{-k_{p}}}{k_{p}^{s_{p}}} Q_{N, p+1}\left(k_{p} ; \underline{z}_{N}^{p+1}\right)
$$

(6) Dans un contexte voisin, Zudilin [49] a introduit une notion de brique, reprise et généralisée dans [23]. Ces briques n'ont rien à voir avec les nôtres; elles sont suffisamment différentes pour ne pas les confondre si on est amené à manipuler les deux types de briques simultanément. 
Enfin, pour tout entier $p$ tel que $2 \leq p \leq N$, on définit

$$
\begin{array}{ll}
R_{\underline{s}_{N}, p}\left(K{ }_{p} \underline{z}_{N}\right) & \\
=\sum_{k_{1} \geq 1} & \frac{z_{1}^{-k_{1}} \cdots z_{p-2}^{-k_{p-2}}\left(z_{p-1} z_{p}\right)^{-k_{p-1}}}{\left(\prod_{i=1}^{p-1}\left(k_{i}+j_{i}\right)^{s_{i}}\right)\left(k_{p-1}+K\right)^{s_{p}}} \\
& \quad \times Q_{N, p+1}\left(k_{p-1}+K ; \underline{z}_{N}^{p+1}\right) . \\
& \vdots \\
k_{1}+m_{2} \geq k_{2} \geq 1 & \\
k_{p-2}+m_{p-1} \geq k_{p-1} \geq 1 &
\end{array}
$$

Si $p=2$, on attribue la valeur 1 au produit vide $z_{1}^{-k_{1}} \cdots z_{p-2}^{-k_{p-2}}$. On notera

$$
R_{\underline{s}_{N}, p}\left(K ; \underline{z}_{N}\right)=R_{N, p}\left(K ; p \underline{z}_{N}\right)
$$

lorsqu'il n'y aura pas de risque de confusion et nous montrerons qu'il s'agit d'un terme de profondeur $\leq N-1$.

4.3. L'algorithme de décomposition des briques. - Le but de ce paragraphe est de démontrer que la brique décalée-modulée (4.3) est la somme de $\left(z_{1}^{j_{1}} \cdots z_{N}^{j_{N}}\right) \operatorname{La}_{\underline{s}_{N}}\left(1 / \underline{z}_{N}\right)$ et de termes de profondeur au plus $N-1$. Cette proposition informelle (qui suffit à démontrer le théorème 1.3, compte tenu des résultats du paragraphe 4.1) découle du théorème suivant qui est beaucoup plus précis.

ThÉorème 4.1. - (i) Pour tout entier $N \geq 1$, on a

$$
\begin{aligned}
\mathrm{B}_{N}\left[\begin{array}{c}
\underline{s}_{N} \\
\underline{m}_{N} \\
\underline{j}_{N}
\end{array} \mid \underline{z}_{N}\right]= & \left(z_{1}^{j_{1}} \cdots z_{N}^{j_{N}}\right) \operatorname{La}_{\underline{s}_{N}}\left(\frac{1}{\underline{z}_{N}}\right) \\
& -\sum_{p=1}^{N}\left(z_{p}^{j_{p}} \cdots z_{N}^{j_{N}}\right) Q_{N, p}\left(j_{p} ; \underline{z}_{N}^{p}\right) \mathrm{B}_{p-1}\left[\begin{array}{c}
\underline{s}_{p-1} \\
\underline{m}_{p-1} \\
\underline{j}_{p-1}
\end{array} \mid \underline{z}_{p}\right] \\
& +\sum_{p=2}^{N} \varepsilon_{p}\left(z_{p}^{j_{p}} \cdots z_{N}^{j_{N}}\right) \sum_{k_{p}=t_{p}+1}^{T_{p}} z_{p}^{-k_{p}} R_{N, p}\left(k_{p} ;_{p} \underline{z}_{N}\right) .
\end{aligned}
$$

(ii) Pour tout entier $p$ tel que $2 \leq p \leq N$ et tout entier $K \geq 0$, la série $R_{N, p}\left(K{ }_{p} \underline{z}_{N}\right)$ est un terme de profondeur $\leq N-1$, dont le poids est inférieur ou égal à $\sum_{p=1}^{N} \max \left(s_{p}, 0\right)$.

Remarque 3. - 1) Si $N=1$, l'expression débutant par $\sum_{p=2}^{N} \varepsilon_{p}(\cdots)$ n'apparaît pas.

2) Ce théorème fournit un algorithme permettant d'expliciter totalement le résultat informel évoqué au début de ce paragraphe. 
Démonstration. - La partie (i) repose sur le lemme suivant, que nous démontrons à la toute fin de ce paragraphe.

Lemme 1. - (i) Pour tout $N \geq 2$ et tout $p=2, \ldots, N$, on a

$$
\begin{aligned}
& \mathrm{B}_{N}\left[\begin{array}{lllll|l}
\underline{s}_{N} & & & \\
\underline{m}_{p} & j_{p} & 0 & \cdots & 0 \\
\underline{j}_{p} & 0 & 0 & \cdots & 0
\end{array} \mid \underline{z}_{N}\right]=z_{p}^{j_{p}} \mathrm{~B}_{N}\left[\begin{array}{lllll}
\underline{s}_{N} & & & \\
\underline{m}_{p-1} & j_{p-1} & 0 & \cdots & 0 \\
\underline{j}_{p-1} & 0 & 0 & \cdots & 0
\end{array} \mid \underline{z}_{N}\right] \\
& -z_{p}^{j_{p}} Q_{N, p}\left(j_{p} ; \underline{z}_{N}^{p}\right) \mathrm{B}_{p-1}\left[\begin{array}{l}
\underline{s}_{p-1} \\
\underline{m}_{p-1} \\
\underline{j}_{p-1}
\end{array} \mid \underline{z}_{p}\right]+\varepsilon_{p} \sum_{k_{p}=t_{p}+1}^{T_{p}} z_{p}^{j_{p}-k_{p}} R_{N, p}\left(k_{p} ;_{p} \underline{z}_{N}\right) .
\end{aligned}
$$

(ii) Pour tout $N \geq 1$, on a

$$
\mathrm{B}_{N}\left[\begin{array}{lllll|l}
\underline{s}_{N} & & & \\
0 & j_{1} & 0 & \cdots & 0 \\
\underline{j}_{1} & 0 & 0 & \cdots & 0
\end{array} \underline{z}_{N}\right]=z_{1}^{j_{1}} \operatorname{La}_{\underline{s}_{N}}\left(\frac{1}{\underline{z}_{N}}\right)-z_{1}^{j_{1}} Q_{N, 1}\left(j_{1} ; \underline{z}_{N}\right) .
$$

On applique le point (i) de ce lemme avec $p=N$, ce qui donne

$$
\begin{aligned}
& \mathrm{B}_{N}\left[\begin{array}{l|l}
\underline{s}_{N} & \underline{m}_{N} \\
\underline{j}_{N} & \underline{z}_{N}
\end{array}\right]=-z_{N}^{j_{N}} Q_{N, N}\left(j_{N} ; z_{N}\right) \mathrm{B}_{N-1}\left[\begin{array}{l}
\underline{s}_{N-1} \\
\underline{m}_{N-1} \\
\underline{j}_{N-1}
\end{array} \underline{z}_{N-1}\right] \\
& +z_{N}^{j_{N}} \mathrm{~B}_{N}\left[\begin{array}{ll}
\underline{s}_{N} & \underline{m}_{N-1} \\
\underline{j}_{N-1} & 0
\end{array} \mid \underline{z}_{N}\right]+\varepsilon_{N} \sum_{k_{N}=t_{N}+1}^{T_{N}} z_{N}^{j_{N}-k_{N}} R_{N, N}\left(k_{N} ;_{N} \underline{z}_{N}\right) .
\end{aligned}
$$

On répète ce procédé $N-1$ fois en appliquant le lemme 1, (i) à l'unique brique de profondeur $N$ qui apparaît à chaque itération, jusqu'à obtenir (en plus d'autres termes) la brique

$$
\mathrm{B}_{N}\left[\begin{array}{llll|l}
\underline{s}_{N} & & & \\
\underline{m}_{1} & j_{1} & 0 & \cdots & 0 \\
\underline{j}_{1} & 0 & 0 & \cdots & 0
\end{array} \mid \underline{z}_{N}\right]
$$

à laquelle on applique alors le point (ii) du même lemme 1 (puisque $\left.\underline{m}_{1}=m_{1}=0\right)$. En regroupant les termes, on constate que l'on a démontré le point (i) du théorème 4.1 .

Pour prouver la partie (ii), on a également besoin d'un lemme technique, dont on donnera la démonstration à la fin du paragraphe. 
Lemme 2. - Soient e, $f \in \mathbb{Z}$ et $i, j \in \mathbb{C}$.

(i) Lorsque $i=j$,

$$
\frac{1}{(X+i)^{e}(X+j)^{f}}=\frac{1}{(X+i)^{e+f}} .
$$

(ii) Lorsque e $\leq 0$ et $f \geq 1$,

$$
\frac{1}{(X+i)^{e}(X+j)^{f}}=\sum_{u=0}^{-e}\left(\begin{array}{c}
-e \\
u
\end{array}\right)(i-j)^{-e-u} \frac{1}{(X+j)^{f-u}} .
$$

(iii) Lorsque e, $f \leq 0$,

$$
\frac{1}{(X+i)^{e}(X+j)^{f}}=\sum_{u=0}^{-e} \sum_{v=0}^{-f}\left(\begin{array}{c}
-e \\
u
\end{array}\right)\left(\begin{array}{c}
-f \\
v
\end{array}\right) i^{-e-u} j^{-f-v} X^{u+v} .
$$

(iv) Lorsque $i \neq j$ et $e, f \geq 1$,

$$
\frac{1}{(X+i)^{e}(X+j)^{f}}=\sum_{u=1}^{e} \frac{\left(\begin{array}{c}
e+f-1-u \\
f-1
\end{array}\right)}{(i-j)^{e+f-u}} \frac{(-1)^{f}}{(X+i)^{u}}+\sum_{v=1}^{f} \frac{\left(\begin{array}{c}
e+f-1-v \\
e-1
\end{array}\right)}{(j-i)^{e+f-v}} \frac{(-1)^{e}}{(X+j)^{v}} .
$$

Nous allons exprimer $R_{N, p}\left(K ;_{p} \underline{z}_{N}\right)$ en termes de briques à l'aide du lemme 2 appliqué à la fraction

$$
\frac{1}{\left(k_{p-1}+j_{p-1}\right)^{s_{p-1}}\left(k_{p-1}+K\right)^{s_{p}}}
$$

qui apparaît dans (4.6). Cinq cas se présentent naturellement et il n'y en a pas d'autres possibles; plusieurs d'entre eux peuvent se produire simultanément mais c'est sans importance ici.

Si $p=N$ (resp. $p=2$ ) les colonnes correspondant à $s_{p+1}, s_{p+2}, \ldots, s_{N}$ (resp. $\underline{s}_{p-2}$ ) des six briques $\mathrm{B}_{N-1}$ suivantes n'apparaissent pas.

4.3.1. Premier cas : $K=j_{p-1}$. - Cela correspond au cas (i) du lemme 2. On a alors

$$
R_{N, p}\left(K ;_{p} \underline{z}_{N}\right)=\mathrm{B}_{N-1}\left[\begin{array}{cccccc}
\underline{s}_{p-2} & s_{p-1}+s_{p} & s_{p+1} & s_{p+2} & \ldots & s_{N} \\
\underline{m}_{p-2} & m_{p-1} & j_{p-1} & 0 & \ldots & 0 \\
\underline{j}_{p-2} & j_{p-1} & 0 & 0 & \ldots & 0
\end{array} \mid \underline{z}_{N}\right]
$$


4.3.2. Deuxième cas : $s_{p-1} \leq 0$ et $s_{p} \geq 1$. - Cela correspond au cas (ii) du lemme 2. On a alors

$$
\begin{aligned}
R_{N, p}\left(K ; p \underline{z}_{N}\right)=\sum_{u=0}^{-s_{p-1}}\left(\begin{array}{c}
-s_{p-1} \\
u
\end{array}\right)\left(j_{p-1}-K\right)^{-s_{p-1}-u} \\
\\
\quad \times \mathrm{B}_{N-1}\left[\left.\begin{array}{cccccc}
\underline{s}_{p-2} & s_{p}-u & s_{p+1} & s_{p+2} & \ldots & s_{N} \\
\underline{m}_{p-2} & m_{p-1} & K & 0 & \ldots & 0 \\
\underline{j}_{p-2} & K & 0 & 0 & \ldots & 0
\end{array}\right|_{p} \underline{z}_{N}\right] .
\end{aligned}
$$

4.3.3. Troisième cas : $s_{p-1} \geq 1$ et $s_{p} \leq 0$. - Cela correspond de nouveau au cas (ii) du lemme 2. On a alors

$$
\begin{aligned}
& R_{N, p}\left(K ;_{p} \underline{z}_{N}\right)=\sum_{u=0}^{-s_{p}}\left(\begin{array}{c}
-s_{p} \\
u
\end{array}\right)\left(K-j_{p-1}\right)^{-s_{p}-u} \\
& \times \mathrm{B}_{N-1}\left[\begin{array}{lccccc}
\underline{s}_{p-2} & s_{p-1}-u & s_{p+1} & s_{p+2} & \ldots & s_{N} \\
\underline{m}_{p-2} & m_{p-1} & K & 0 & \ldots & 0 \\
\underline{j}_{p-2} & j_{p-1} & 0 & 0 & \ldots & 0
\end{array} \mid \underline{z}_{N}\right] .
\end{aligned}
$$

4.3.4. Quatrième cas : $s_{p-1} \leq 0$ et $s_{p} \leq 0$. - Cela correspond au cas (iii) du lemme 2. On a alors

$$
\begin{aligned}
& R_{N, p}\left(K ; p \underline{z}_{N}\right)=\sum_{u=0}^{-s_{p-1}} \sum_{v=0}^{-s_{p}}\left(\begin{array}{c}
-s_{p-1} \\
u
\end{array}\right)\left(\begin{array}{c}
-s_{p} \\
v
\end{array}\right) j_{p-1}^{-s_{p-1}-u} K^{-s_{p}-v} \\
& \times \mathrm{B}_{N-1}\left[\begin{array}{cccccc}
\underline{s}_{p-2} & -u-v & s_{p+1} & s_{p+2} & \ldots & s_{N} \\
\underline{m}_{p-2} & m_{p-1} & K & 0 & \ldots & 0 \\
\underline{j}_{p-2} & 0 & 0 & 0 & \ldots & 0
\end{array} \mid \underline{z}_{N}\right]
\end{aligned}
$$


4.3.5. Cinquième cas : $K \neq j_{p-1}, s_{p-1} \geq 1$ et $s_{p} \geq 1$. - Cela correspond au cas (iv) du lemme 2. On a alors

$$
\begin{aligned}
& R_{N, p}\left(K{ }_{p} \underline{z}_{N}\right) \\
& =(-1)^{s_{p}} \sum_{u=1}^{s_{p-1}} \frac{\left(\begin{array}{c}
s_{p-1}+s_{p}-1-u \\
s_{p}-1
\end{array}\right)}{\left(j_{p-1}-K\right)^{s_{p-1}+s_{p}-u}} \\
& \times \mathrm{B}_{N-1}\left[\begin{array}{lccccc}
\underline{s}_{p-2} & u & s_{p+1} & s_{p+2} & \ldots & s_{N} \\
\underline{m}_{p-2} & m_{p-1} & K & 0 & \ldots & 0 \\
\underline{j}_{p-2} & j_{p-1} & 0 & 0 & \ldots & 0
\end{array} p \underline{z}_{N}\right] \\
& +(-1)^{s_{p-1}} \sum_{v=1}^{s_{p}} \frac{\left(\begin{array}{c}
s_{p-1}+s_{p}-1-v \\
s_{p-1}-1
\end{array}\right)}{\left(K-j_{p-1}\right)^{s_{p-1}+s_{p}-v}} \\
& \times \mathrm{B}_{N-1}\left[\begin{array}{lccccc}
\underline{s}_{p-2} & v & s_{p+1} & s_{p+2} & \ldots & s_{N} \\
\underline{m}_{p-2} & m_{p-1} & K & 0 & \ldots & 0 \\
\underline{j}_{p-2} & K & 0 & 0 & \ldots & 0
\end{array} \mid \underline{z}_{N}\right]
\end{aligned}
$$

Chacun de ces cinq cas montre que $R_{N, p}\left(K{ }_{p} \underline{z}_{N}\right)$ est un terme de profondeur $\leq N-1$, de poids $\leq \sum_{p=1}^{N} \max \left(s_{p}, 0\right)$, ce qui conclut la preuve.

Démonstration du lemme 1. - Montrons (i). Remarquons tout d'abord que pour toute suite $\left(u_{n}\right)_{n \geq 0}$, on a

$$
\begin{aligned}
& \sum_{\ell=1}^{k+m} \frac{u_{\ell+j} z^{-\ell}}{(\ell+j)^{s}}=\sum_{\ell=j+1}^{k+j+m} \frac{u_{\ell} z^{j-\ell}}{\ell^{s}}
\end{aligned}
$$

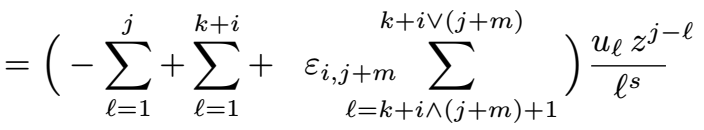

$$
\begin{aligned}
& =\left(-\sum_{\ell=1}^{j}+\sum_{\ell=1}^{k+i}\right) \frac{u_{\ell} z^{j-\ell}}{\ell^{s}}+\varepsilon_{i, j+m} \sum_{\ell=i \wedge(j+m)+1}^{i \vee(j+m)} \frac{u_{k+\ell} z^{j-k-\ell}}{(k+\ell)^{s}},
\end{aligned}
$$

après quelques manipulations immédiates. 
Supposons maintenant $2 \leq p \leq N-1$. On a

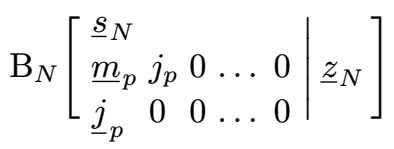

$$
\begin{aligned}
& =\sum_{k_{1} \geq 1} \frac{z_{1}^{-k_{1}} \cdots z_{p}^{-k_{p-1}}}{\prod_{i=1}^{p-1}\left(k_{i}+j_{i}\right)^{s_{i}}} \sum_{k_{p}=1}^{k_{p-1}+m_{p}} \frac{z_{p}^{-k_{p}}}{\left(k_{p}+j_{p}\right)^{s_{p}}} Q_{N, p+1}\left(k_{p}+j_{p} ; \underline{z}_{N}^{p+1}\right) \text {. } \\
& k_{1}+m_{2} \geq k_{2} \geq 1 \\
& \text { : } \\
& k_{p-2}+m_{p-1} \geq k_{p-1} \geq 1
\end{aligned}
$$

On applique (4.8) à la somme $\sum_{k_{p}=1}^{k_{p-1}+m_{p}}(\ldots)$ et à la suite $u_{n}=Q_{N, p+1}\left(n ; \underline{x}_{N}^{p+1}\right)$ : grâce à la relation (4.5) entre $Q_{N, p}$ et $Q_{N, p+1}$, on voit alors que

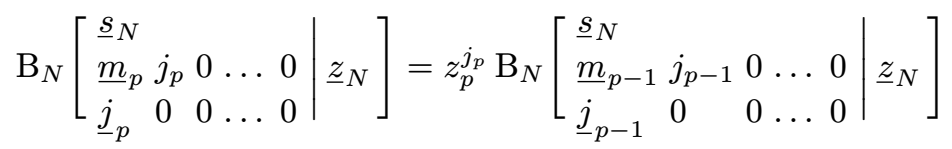

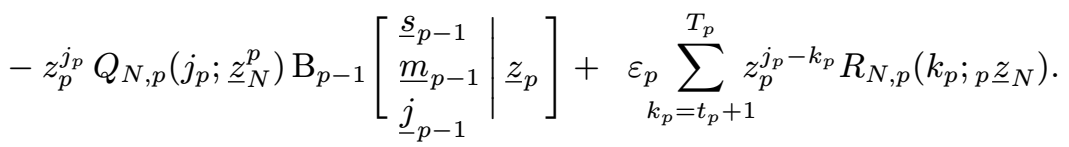

Pour (ii), on a

$$
\begin{gathered}
\mathrm{B}_{N}\left[\begin{array}{lllll|l}
\underline{s}_{N} & & & \\
0 & j_{1} & 0 & \ldots & 0 & \underline{z}_{N} \\
\underline{j}_{1} & 0 & 0 & \ldots & 0
\end{array}\right]=\sum_{k_{1}=1}^{\infty} \frac{z_{1}^{-k_{1}}}{\left(k_{1}+j_{1}\right)^{s_{1}}} Q_{N, 2}\left(k_{1}+j_{1} ; \underline{z}_{N}^{2}\right) \\
\quad=\sum_{k_{1}=j_{1}+1}^{\infty} \frac{z_{1}^{j_{1}-k_{1}}}{k_{1}^{s_{1}}} Q_{N, 2}\left(k_{1} ; \underline{z}_{N}^{2}\right) \\
=z_{1}^{j_{1}} \mathrm{~B}_{N}\left[\begin{array}{llll|l}
\underline{s}_{N} & 0 & 0 & 0 \\
0 & 0 & 0 & \ldots & 0
\end{array}\right]-\sum_{k_{1}=1}^{j_{1}} \frac{z_{1}^{j_{1}-k_{1}}}{k_{1}^{s_{1}}} Q_{N, 2}\left(k_{1} ; \underline{z}_{N}^{2}\right) \\
=z_{1}^{j_{1}} \mathrm{La}_{\underline{s}_{N}}\left(\frac{1}{\underline{z}_{N}}\right)-z_{1}^{j_{1}} Q_{N, 1}\left(j_{1} ; \underline{z}_{N}\right)
\end{gathered}
$$

ce qui termine la démonstration du lemme. 
Démonstration du lemme 2. - Les points (i), (ii) et (iii) sont triviaux et on démontre seulement (iv), qui l'est à peine moins. En effet, on a

$$
\frac{1}{(X+i)^{e}(X+j)^{f}}=\sum_{u=1}^{e} \frac{a_{u}}{(X+i)^{u}}+\sum_{v=1}^{f} \frac{b_{v}}{(X+j)^{v}}
$$

avec

$$
\begin{aligned}
a_{u} & =\left.\frac{1}{(e-u) !}\left(\frac{1}{(X+j)^{f}}\right)^{e-u}\right|_{X=-i} \\
& =\frac{(-f)(-f-1) \cdots(-f-e+u+1)}{(e-u) !(j-i)^{e+f-u}} \\
& =\left(\begin{array}{c}
e+f-u-1 \\
f-1
\end{array}\right) \frac{(-1)^{f}}{(i-j)^{e+f-u}}
\end{aligned}
$$

et la formule similaire attendue pour $b_{v}$.

\section{Précisions sur le théorème 4.1}

Le but de ce paragraphe est de préciser la nature des polynômes de Laurent qui apparaissent quand on itère le théorème 4.1, sous la condition que tous les exposants $s_{i}$ sont strictement positifs.

On pose :

- $M_{i}=\sum_{k=1}^{i} m_{k}$ avec $M_{0}=0$;

- $I_{N}=\max _{i=1, \ldots, N}\left(T_{i}+M_{i-1}\right)$ avec $T_{i}=j_{i-1} \vee\left(j_{i}+m_{i}\right), j_{0}=0$ et $I_{0}=0$;

- $J_{N}=\max _{i=1, \ldots, N}\left(j_{i}\right)$ et $J_{0}=0$;

- $K_{N}=\max _{i=1, \ldots, N}\left(T_{i}\right)$ et $K_{0}=0$;

- $\Sigma_{N}=\sum_{i=1}^{N} s_{i}$.

$J_{N}$ est le cas spécial de $I_{N}$ obtenu lorsque les modulations sont toutes nulles. Rappelons que $\mathrm{d}_{n}$ désigne le ppcm des entiers $1,2, \ldots, n$. Par convention, $\mathrm{d}_{0}=1$. On utilisera le fait trivial que $\mathrm{d}_{n}^{e} \mathrm{~d}_{m}^{f}$ divise $\mathrm{d}_{n \vee m}^{e+f}$.

THÉORÈME 5.1. - Supposons que tous les exposants $s_{i}$ sont strictement positifs.

(i) Les polynômes de Laurent qui interviennent dans la décomposition de la brique décalée-modulée large (4.3) en polylogarithmes multiples sont dans

$$
d_{I_{N}}^{-\Sigma_{N}} \mathbb{Z}\left[z_{1}, z_{2}^{ \pm 1}, \ldots, z_{N}^{ \pm 1}\right]
$$

et leur degré en $z_{1}$ est au plus $K_{N}$.

(ii) Les polynômes de Laurent qui interviennent dans la décomposition de la brique décalée large (4.4) en polylogarithmes multiples sont dans

$$
d_{J_{N}}^{-\Sigma_{N}} \mathbb{Z}\left[z_{1}, z_{2}^{ \pm 1}, \ldots, z_{N}^{ \pm 1}\right]
$$

TOME $136-2008-\mathrm{N}^{\circ} 1$ 
et leur degré en $z_{1}$ est au plus $J_{N}$.

REMARQue 4. - 1) Le point (ii) est le seul vraiment utile; nous ne savons pas le démontrer sans d'abord démontrer (i), dont il est un cas particulier.

2) On n'utilisera pas que les $s_{i}$ sont strictement positifs pour démontrer que les polynômes de Laurent sont des polynômes de degré $\leq j_{1}$ en la variable $z_{1}$.

3) Concernant le dénominateur, un résultat similaire a probablement lieu dans le cas général mais nous n'avons pas cherché à l'expliciter, faute de perspectives diophantiennes évidentes.

Démonstration. - (i) Nous procédons, en deux temps, par récurrence sur la profondeur $N$ de la brique (4.3) : le point (ii) en découle en prenant le cas particulier de modulations toutes nulles.

5.1. Preuve de l'assertion sur les dénominateurs. - Le cas $N=1$ est immédiat : on a

$$
\mathrm{B}_{1}\left[\begin{array}{c|c}
s_{1} & \\
0 & z_{1} \\
j_{1} &
\end{array}\right]=z_{1}^{j_{1}} \operatorname{La}_{s_{1}}\left(z_{1}\right)-z_{1}^{j_{1}} Q_{1,1}\left(j_{1} ; z_{1}\right)
$$

où $Q_{1,1}\left(j_{1} ; z_{1}\right)=\sum_{k_{1}=1}^{j_{1}} \frac{z_{1}^{-k_{1}}}{k_{1}^{s_{1}}}$ a pour dénominateur $\mathrm{d}_{j_{1}}^{s_{1}}=\mathrm{d}_{I_{1}}^{\Sigma_{1}}$.

Supposons maintenant le théorème 5.1 vrai jusqu'à la profondeur $N-1$ et analysons les différents termes de l'équation (4.7), que nous rappelons :

$$
\begin{aligned}
& \mathrm{B}_{N}\left[\begin{array}{l|l}
\underline{s}_{N} & \\
\underline{m}_{N} & \underline{z}_{N} \\
\underline{j}_{N}
\end{array}\right]=\left(z_{1}^{j_{1}} \cdots z_{N}^{j_{N}}\right) \operatorname{La}_{\underline{s}_{N}}\left(\underline{z}_{N}\right) \\
& -\sum_{p=1}^{N}\left(z_{p}^{j_{p}} \cdots z_{N}^{j_{N}}\right) Q_{N, p}\left(j_{p} ; \underline{z}_{N}^{p}\right) \mathrm{B}_{p-1}\left[\begin{array}{l}
\underline{s}_{p-1} \\
\underline{m}_{p-1} \\
\underline{j}_{p-1}
\end{array} \mid \underline{z}_{p}\right] \\
& +\sum_{p=2}^{N} \varepsilon_{p}\left(z_{p}^{j_{p}} \cdots z_{N}^{j_{N}}\right) \sum_{k_{p}=t_{p}+1}^{T_{p}} z_{p}^{-k_{p}} R_{N, p}\left(k_{p} ; p \underline{z}_{N}\right) .
\end{aligned}
$$

Tout d'abord

$$
Q_{N, p}\left(j_{p} ; \underline{z}_{N}^{p}\right)=\sum_{j_{p} \geq k_{p} \geq \cdots \geq k_{N} \geq 1} \frac{z_{p}^{-k_{p}} \cdots z_{N}^{-k_{N}}}{\prod_{i=p}^{N} k_{i}^{s_{i}}}
$$


a pour dénominateur $\mathrm{d}_{j_{p}}^{s_{p}+\cdots+s_{N}}$. Par hypothèse de récurrence, un dénominateur de la brique $\mathrm{B}_{p-1}$ est $\mathrm{d}_{I_{p-1}}^{s_{1}+\cdots+s_{p-1}}$, même pour $p=1$. Un dénominateur des termes $Q_{N, p} \mathrm{~B}_{p-1}$ est donc $\mathrm{d}_{j_{p}}^{s_{p}+\cdots+s_{N}} \mathrm{~d}_{I_{p-1}}^{s_{1}+\cdots+s_{p-1}}$, qui divise $\mathrm{d}_{I_{N}}^{\Sigma_{N}}$ puisque

$$
j_{p} \vee I_{p-1} \leq\left(T_{p}+M_{p-1}\right) \vee I_{p-1}=I_{p} \leq I_{N}
$$

pour tout $p \in\{1, \ldots, N\}$.

Il reste à analyser les termes $R_{N, p}\left(k_{p} ;_{p} \underline{z}_{N}\right)$ : nous allons distinguer deux cas.

5.1.1. Premier cas : $k_{p}=j_{p-1}$. - On est alors dans la situation du paragraphe 4.3.1 :

$$
R_{N, p}\left(j_{p-1} ;{ }_{p} \underline{z}_{N}\right)=\mathrm{B}_{N-1}\left[\begin{array}{cccccc}
\underline{s}_{p-2} & s_{p-1}+s_{p} & s_{p+1} & s_{p+2} & \cdots & s_{N} \\
\underline{m}_{p-2} & m_{p-1} & j_{p-1} & 0 & \cdots & 0 \\
\underline{j}_{p-2} & j_{p-1} & 0 & 0 & \cdots & 0
\end{array} \mid \underline{z}_{N}\right] .
$$

L'hypothèse de récurrence s'applique : un dénominateur de la brique est

$$
\mathrm{d}_{I_{p-1} \vee\left(j_{p-1} \vee\left(0+j_{p-1}\right)+M_{p}\right)}^{\Sigma_{N}}=\mathrm{d}_{I_{p-1} \vee\left(j_{p-1}+M_{p}\right)}^{\Sigma_{N}} .
$$

Comme $j_{p-1}+M_{p-1} \leq T_{p}+M_{p-1}$, ce dénominateur divise $\mathrm{d}_{I_{p}}^{\Sigma_{N}}$, qui divise $\mathrm{d}_{I_{N}}^{\Sigma_{N}}$.

5.1.2. Second cas : $k_{p} \neq j_{p-1}$. - On est maintenant dans la situation du paragraphe 4.3 .5 :

$$
\begin{aligned}
\sum_{p=2}^{N} \varepsilon_{p}\left(z_{p}^{j_{p}} \cdots z_{N}^{j_{N}}\right) \sum_{\substack{k_{p}=t_{p}+1 \\
k_{p} \neq j_{p-1}}}^{T_{p}} R_{N, p}\left(k_{p} ; p \underline{z}_{N}\right) \\
=\sum_{p=2}^{N} \varepsilon_{p}\left(z_{p}^{j_{p}} \cdots z_{N}^{j_{N}}\right)\left(\sum_{u=1}^{s_{p-1}} B_{1, p}(u)+\sum_{v=1}^{s_{p}} B_{2, p}(v)\right)
\end{aligned}
$$

avec

$$
\begin{aligned}
B_{1, p}(u)=(-1)^{s_{p}} & \sum_{\substack{k_{p}=t_{p}+1 \\
k_{p} \neq j_{p-1}}}^{T_{p}} \frac{\left(\begin{array}{c}
s_{p-1}+s_{p}-1-v \\
s_{p}-1
\end{array}\right)}{\left(j_{p-1}-k_{p}\right)^{s_{p-1}+s_{p}-u}} \\
& \times \mathrm{B}_{N-1}\left[\begin{array}{cccccc}
\underline{s}_{p-2} & u & s_{p+1} & s_{p+2} & \cdots & s_{N} \\
\underline{m}_{p-2} & m_{p-1} & k_{p} & 0 & \cdots & 0 \\
\underline{j}_{p-2} & j_{p-1} & 0 & 0 & \cdots & 0
\end{array} \mid \underline{z}_{N}\right]
\end{aligned}
$$


et

$$
\begin{aligned}
B_{2, p}(v)=(-1)^{s_{p-1}} & \sum_{\substack{k_{p}=t_{p}+1 \\
k_{p} \neq j_{p-1}}}^{T_{p}} \frac{\left(\begin{array}{c}
s_{p-1}+s_{p}-1-v \\
s_{p-1}-1
\end{array}\right)}{\left(k_{p}-j_{p-1}\right)^{s_{p-1}+s_{p}-v}} \\
& \times \mathrm{B}_{N-1}\left[\left.\begin{array}{llllll}
\underline{s}_{p-2} & v & s_{p+1} & s_{p+2} & \cdots & s_{N} \\
\underline{m}_{p-2} & m_{p-1} & k_{p} & 0 & \cdots & 0 \\
\underline{j}_{p-2} & k_{p} & 0 & 0 & \cdots & 0
\end{array}\right|_{p} \underline{z}_{N}\right] .
\end{aligned}
$$

Nous allons montrer que $\mathrm{d}_{I_{N}}^{\Sigma_{N}}$ est un dénominateur convenable pour les termes (5.2) et (5.3), ce qui suffira puisqu'il est indépendant de $p, u$ et $v$. Fixons $p, u$ et $v$. Par hypothèse de récurrence, les deux briques $\mathrm{B}_{N-1}$ ont pour dénominateurs respectifs

$$
D_{1}=\mathrm{d}_{I_{p-1} \vee\left(k_{p}+M_{p-1}\right)}^{u+\Sigma_{N}-s_{p}-s_{p-1}} \quad \text { et } \quad D_{2}=\mathrm{d}_{I_{p-2} \vee\left(j_{p-2} \vee\left(k_{p}+m_{p-1}\right)+M_{p-2}\right) \vee\left(k_{p}+M_{p-1}\right)}^{v+\Sigma_{N}-s_{p}-s_{p-1}} .
$$

Puisque $k_{p} \leq T_{p}$, on a

$$
I_{p-1} \vee\left(k_{p}+M_{p-1}\right) \leq I_{p-1} \vee\left(T_{p}+M_{p-1}\right)=I_{p}
$$

et donc $D_{1}$ divise $\mathrm{d}_{I_{p}}^{u+\Sigma_{N}-s_{p}-s_{p-1}}$. D'autre part, si $j_{p-2} \leq k_{p}+m_{p-1}$, on a

$$
j_{p-2} \vee\left(k_{p}+m_{p-1}\right)+M_{p-2} \leq k_{p}+m_{p-1}+M_{p-2} \leq T_{p}+M_{p-1}
$$

tandis que si $j_{p-2} \geq k_{p}+m_{p-1}$, alors

$$
j_{p-2} \vee\left(k_{p}+m_{p-1}\right)+M_{p-2} \leq j_{p-2}+M_{p-2} \leq T_{p-1}+M_{p-2},
$$

d'où $D_{2}$ divise $\mathrm{d}_{I_{p-2} \vee\left(T_{p-1}+M_{p-2}\right) \vee\left(T_{p}+M_{p-1}\right)}^{v+\Sigma_{N}-s_{p}-s_{p-1}}=\mathrm{d}_{I_{p}}^{v+\Sigma_{N}-s_{p}-s_{p-1}}$. On obtient donc des dénominateurs uniformes en $k_{p}$ pour les briques $\mathrm{B}_{N-1}$ :

$$
\mathrm{d}_{I_{p}}^{u+\Sigma_{N}-s_{p}-s_{p-1}} \quad \text { et } \mathrm{d}_{I_{p}}^{v+\Sigma_{N}-s_{p}-s_{p-1}} .
$$

Les deux sommes

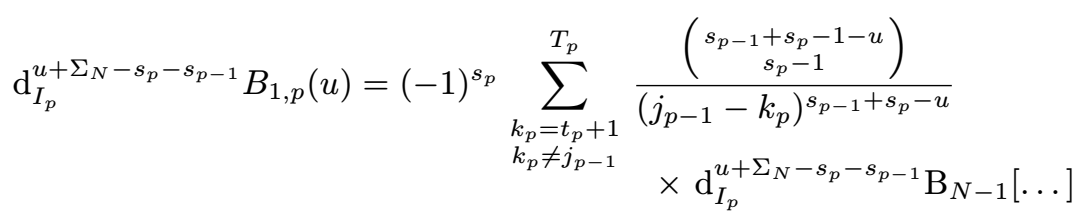

et

$$
\begin{aligned}
& \mathrm{d}_{I_{p}}^{v+\Sigma_{N}-s_{p}-s_{p-1}} B_{2, p}(v)=(-1)^{s_{p-1}} \sum_{k_{p}=t_{p}+1}^{T_{p}} \frac{\left(\begin{array}{c}
s_{p-1}+s_{p}-1-v \\
s_{p-1}-1
\end{array}\right)}{\left(k_{p}-j_{p-1}\right)^{s_{p-1}+s_{p}-v}} \\
& k_{p} \neq j_{p-1} \times \mathrm{d}_{I_{p}}^{v+\Sigma_{N}-s_{p}-s_{p-1}} \mathrm{~B}_{N-1}[\ldots]
\end{aligned}
$$

admettent donc pour dénominateurs respectifs

$$
\mathrm{d}_{\left|j_{p}+m_{p}-j_{p-1}\right|}^{-u+s_{p-1}+s_{p}} \quad \text { et } \quad \mathrm{d}_{\left|j_{p}+m_{p}-j_{p-1}\right|}^{-v+s_{p-1}+s_{p}},
$$

BULLETIN DE LA SOCIÉTÉ MATHÉMATIQUE DE FRANCE 
qui divisent trivialement $\mathrm{d}_{I_{p}}^{-u+s_{p-1}+s_{p}}$, respectivement $\mathrm{d}_{I_{p}}^{-v+s_{p-1}+s_{p}}$, car

$$
\left|j_{p}+m_{p}-j_{p-1}\right| \leq T_{p} \leq I_{p}
$$

Ainsi, on peut prendre

$$
\mathrm{d}_{I_{p}}^{-u+s_{p-1}+s_{p}} \mathrm{~d}_{I_{p}}^{u+\Sigma_{N}-s_{p}-s_{p-1}}=\mathrm{d}_{I_{p}}^{\Sigma_{N}}, \quad \mathrm{~d}_{I_{p}}^{-v+s_{p-1}+s_{p}} \mathrm{~d}_{I_{p}}^{v+\Sigma_{N}-s_{p}-s_{p-1}}=\mathrm{d}_{I_{p}}^{\Sigma_{N}},
$$

comme dénominateurs de (5.2) et (5.3), ce qui achève la preuve du théorème 5.1 puisque $\mathrm{d}_{I_{p}}^{\Sigma_{N}}$ divise $\mathrm{d}_{I_{N}}^{\Sigma_{N}}$.

5.2. Preuve de l'assertion sur le degré en $z_{1}$. - De nouveau, on raisonne par récurrence sur la profondeur $N \geq 1$. C'est évidemment vrai pour $N=1$ par l'équation (5.1). Supposons maintenant l'assertion vraie pour $N-1$ et, comme précédemment, analysons les termes de l'équation (4.7). Le terme $\left(z_{1}^{j_{1}} \cdots z_{N}^{j_{N}}\right) \operatorname{La}_{\underline{s}_{N}}\left(\underline{z}_{N}\right)$ est de la forme voulue, avec un degré $j_{1} \leq K_{N}$. Dans le terme

$$
\sum_{p=1}^{N}\left(z_{p}^{j_{p}} \cdots z_{N}^{j_{N}}\right) Q_{N, p}\left(j_{p} ; \underline{z}_{N}^{p}\right) \mathrm{B}_{p-1}\left[\begin{array}{l}
\underline{s}_{p-1} \\
\underline{m}_{p-1} \\
\underline{j}_{p-1}
\end{array} \mid \underline{z}_{p}\right],
$$

si $p \geq 2$, la variable $z_{1}$ n'apparaît pas dans les polynômes de Laurent $Q_{N, p}\left(j_{p} ; \underline{z}_{N}^{p}\right)$ et seulement dans la brique $B_{p-1}[\ldots]$ qui est de profondeur $p-1 \leq N-1$ : l'hypothèse de récurrence s'applique et seules les puissances positives de $z_{1}$ interviennent bien, jusqu'au plus $z_{1}^{K_{p-1}}$, donc au plus $z_{1}^{K_{N}}$. Si $p=1$, alors $z_{1}$ intervient dans l'expression

$$
z_{1}^{j_{1}} Q_{N, 1}\left(j_{1} ; \underline{z}_{N}^{1}\right) \mathrm{B}_{0}[\ldots]=z_{1}^{j_{1}} Q_{N, 1}\left(j_{1} ; \underline{z}_{N}\right),
$$

qui est aussi un polynôme en $z_{1}$ de degré au plus $j_{1} \leq K_{N}$. Il reste le dernier terme

$$
\sum_{p=2}^{N} \varepsilon_{p}\left(z_{p}^{j_{p}} \cdots z_{N}^{j_{N}}\right) \sum_{k_{p}=t_{p}+1}^{T_{p}} z_{p}^{-k_{p}} R_{N, p}\left(k_{p} ; \underline{z}_{N}\right)
$$

qui ne dépend de $z_{1}$ que par $R_{N, p}\left(k_{p} ;{ }_{p} \underline{z}_{N}\right)$. Or les expressions que nous en avons données au paragraphe précédent montrent qu'il s'agit d'une combinaison linéaire de briques de profondeur $\leq N-1$ évaluées en $\underline{z}_{N}$ et dont les coefficients ne dépendent pas des $z_{i}$. Dans ${ }_{p} \underline{z}_{N}$, la variable $z_{1}$ apparaît seule si $3 \leq p \leq N$ : l'hypothèse de récurrence s'applique et on vérifie que le degré en $z_{1}$ est au plus $K_{p} \leq K_{N}$. Si $p=2$, alors il y a une subtilité car $z_{1}$ apparaît multiplié par $z_{2}$ : ce n'est pas gênant, l'hypothèse de récurrence s'applique de nouveau et le degré en $z_{1}$ est $\leq T_{2} \leq K_{N}$, ce qui conclut la démonstration. 


\section{Non-enrichissement des $\mathrm{La}_{s_{1}, \ldots, s_{p}}$ à exposants négatifs}

L'algorithme de décomposition des briques peut faire apparaître des polylogarithmes larges à exposants négatifs (ou nuls). Par exemple la décomposition de l'intégrale de Sorokin pour $\zeta(3)$

$$
\int_{[0,1]^{3}} \frac{u^{n}(1-u)^{n} v^{n}(1-v)^{n} w^{n}(1-w)^{n}}{\left(z_{1}-u v\right)^{n+1}\left(z_{1} z_{2}-u v w\right)^{n+1}} \mathrm{~d} u \mathrm{~d} v \mathrm{~d} w
$$

fait intervenir des $\operatorname{La}_{s_{1}, s_{2}}\left(1 / z_{1}, 1 / z_{2}\right)$, avec $s_{1}=1,2, s_{2}=0,-1, \ldots,-n+1$.

Afin de régler ces cas singuliers, on démontre un résultat dit de nonenrichissement arithmétique.

THÉORÈme 6.1. - Supposons que, pour tout $j=1, \ldots, p$, on ait $\left|z_{j}\right|<1$. Alors, tout polylogarithme multiple large $\operatorname{La}_{s_{1}, \ldots, s_{p}}\left(z_{1}, \ldots, z_{p}\right)$ de profondeur $p$ ayant certains exposants $s_{j} \leq 0$ s'exprime comme une combinaison linéaire finie de polylogarithmes multiples larges $\operatorname{La}_{s_{1}^{\prime}, \ldots, s_{q}^{\prime}}\left(z_{1}^{*}, \ldots, z_{q}^{*}\right)$ de profondeur $q \in\{0, \ldots, p\}$, avec $s_{j}^{\prime} \geq 1$, où les $z_{i}^{*}$ sont certains produits des $z_{j}$. Les coefficients de la combinaison linéaire sont des polynômes à coefficients rationnels en les

$$
\left(\left(1-z_{j_{1}} \cdots z_{j_{m}}\right)^{-1}\right)_{1 \leq j_{1}<\cdots<j_{m} \leq p, m \geq 1} \quad \text { et les } \quad\left(z_{j}^{ \pm 1}\right)_{1 \leq j \leq p}
$$

De plus, on a

$$
\sum_{j=1}^{q} s_{j}^{\prime} \leq \sum_{j=1}^{p} \max \left(0, s_{j}\right)
$$

pour toutes les suites d'exposants $\underline{s}^{\prime}$ qui apparaissent.

Remarque 5. - 1) Pour tout $z$ tel que $|z|<1$, on a

$$
\operatorname{La}_{-s}(z)=\left(z \frac{\mathrm{d}}{\mathrm{d} z}\right)^{s}\left(\frac{1}{1-z}\right) \in(1-z)^{-s-1} \mathbb{Z}[z] .
$$

2) Ce théorème est de facture informelle mais sa démonstration offre un moyen algorithmique de l'expliciter.

3) Un résultat de ce type est annoncé par Écalle [13, p. 419-420] dans le cas des polyzêtas, sans démonstration. 
6.1. Préliminaires. - On suppose dans toute la suite de ce paragraphe que toutes les variables notées $z$ ou $z_{j}$ sont de modules $<1$. La démonstration utilisera l'identité triviale suivante, valable pour tout entier $K \geq 1$ :

$$
\sum_{k_{1}=1}^{K} \sum_{k_{2}=1}^{k_{1}}=\sum_{k_{2}=1}^{K}\left(\sum_{k_{1}=1}^{K}-\sum_{k_{1}=1}^{k_{2}-1}\right)
$$

Pour tous entiers $s \geq 0$ et $K \geq 1$, on définit $P_{s}(K, z)=\sum_{k=1}^{K} k^{s} z^{k}$, qui vérifie

$$
P_{s}(K, z)=\left(z \frac{\mathrm{d}}{\mathrm{d} z}\right)^{s}\left(z \frac{1-z^{K}}{1-z}\right) \text {. }
$$

On en déduit que l'on a

$$
P_{s}(K, z)=\sum_{\ell=0}^{s} \frac{z^{K} a_{1, \ell}(s, z)+a_{2, \ell}(s, z)}{(1-z)^{s+1}} K^{\ell}
$$

où $a_{1, \ell}(s, z)$ et $a_{2, \ell}(s, z)$ sont des polynômes en $z$ de degré au plus $s$ et indépendants de $K$.

Les objets naturels qui vont intervenir sont des polylogarithmes larges tronqués :

$$
\mathrm{La}_{s_{1}, \ldots, s_{p}}^{K}\left(z_{1}, \ldots, z_{p}\right)=\sum_{K \geq k_{1} \geq \cdots \geq k_{p} \geq 1} \frac{z_{1}^{k_{1}} \cdots z_{p}^{k_{p}}}{k_{1}^{s_{1}} \cdots k_{p}^{s_{p}}}
$$

On remarque que l'on a $\operatorname{La}_{s_{1}}^{K}\left(z_{1}\right)=P_{-s_{1}}\left(K, z_{1}\right)$ lorsque $s_{1} \leq 0$.

On aura besoin du lemme suivant.

Lemme 3. - Soient des entiers $s_{1} \geq 0$ et $s_{2}, \ldots, s_{p} \in \mathbb{Z}$. Pour tous entiers $K \geq 1$ et $p \geq 2$, on $a$

$$
\begin{aligned}
& \mathrm{La}_{-s_{1}, s_{2}, \ldots, s_{p}}^{K}\left(z_{1}, \ldots, z_{p}\right)=P_{s_{1}}\left(K, z_{1}\right) \mathrm{La}_{s_{2}, \ldots, s_{p}}^{K}\left(z_{2}, \ldots, z_{p}\right) \\
& \quad-\sum_{\ell=0}^{s_{1}} \frac{a_{1, \ell}\left(s_{1}, z_{1}\right)}{\left(1-z_{1}\right)^{s_{1}+1} z_{1}} \sum_{m=0}^{\ell}\left(\begin{array}{c}
\ell \\
m
\end{array}\right)(-1)^{\ell-m} \operatorname{La}_{s_{2}-m, s_{3}, \ldots, s_{p}}^{K}\left(z_{1} z_{2}, z_{3}, \ldots, z_{p}\right) \\
& \quad-\sum_{\ell=0}^{s_{1}} \frac{a_{2, \ell}\left(s_{1}, z_{1}\right)}{\left(1-z_{1}\right)^{s_{1}+1}} \sum_{m=0}^{\ell}\left(\begin{array}{c}
\ell \\
m
\end{array}\right)(-1)^{\ell-m} \operatorname{La}_{s_{2}-m, s_{3}, \ldots, s_{p}}^{K}\left(z_{2}, \ldots, z_{p}\right) .
\end{aligned}
$$

TOME $136-2008-\mathrm{N}^{\mathrm{O}} 1$ 
Démonstration. - En utilisant (6.1), on a

$$
\begin{array}{r}
\mathrm{La}_{-s_{1}, s_{2}, \ldots, s_{p}}^{K}\left(z_{1}, \ldots, z_{p}\right)=\sum_{k_{2}=1}^{K} \frac{z_{2}^{k_{2}}}{k_{2}^{s_{2}}}\left(\sum_{k_{1}=1}^{K} k_{1}^{s_{1}} z^{k_{1}}-\sum_{k_{1}=1}^{k_{2}-1} k_{1}^{s_{1}} z^{k_{1}}\right) \\
\times \operatorname{La}_{s_{3}, \ldots, s_{p}}^{k_{2}}\left(z_{3}, \ldots, z_{p}\right) \\
=\sum_{k_{2}=1}^{K} \frac{z_{2}^{k_{2}}}{k_{2}^{s_{2}}}\left(P_{s_{1}}\left(K, z_{1}\right)-P_{s_{1}}\left(k_{2}-1, z_{1}\right)\right) \\
\times \operatorname{La}_{s_{3}, \ldots, s_{p}}^{k_{2}}\left(z_{3}, \ldots, z_{p}\right) .
\end{array}
$$

Au moyen de (6.2), on obtient

$$
\begin{aligned}
& \operatorname{La}_{-s_{1}, s_{2}, \ldots, s_{p}}^{K}\left(z_{1}, \ldots, z_{p}\right)=P_{s_{1}}\left(K, z_{1}\right) \sum_{k_{2}=1}^{K}\left(\frac{z_{2}^{k_{2}}}{k_{2}^{s_{2}}} \operatorname{La}_{s_{3}, \ldots, s_{p}}^{k_{2}}\left(z_{3}, \ldots, z_{p}\right)\right) \\
& -\sum_{\ell=0}^{s_{1}} \frac{1}{\left(1-z_{1}\right)^{s_{1}+1}} \sum_{k_{2}=1}^{K}\left(z_{1}^{k_{2}-1} a_{1, \ell}\left(s_{1}, z_{1}\right)+a_{2, \ell}\left(s_{1}, z_{1}\right)\right) \\
& \times\left(\frac{z_{2}^{k_{2}}}{k_{2}^{s_{2}}}\left(k_{2}-1\right)^{\ell} \operatorname{La}_{s_{3}, \ldots, s_{p}}^{k_{2}}\left(z_{3}, \ldots, z_{p}\right)\right) .
\end{aligned}
$$

La première somme vaut exactement

$$
P_{s_{1}}\left(K, z_{1}\right) \operatorname{La}_{s_{2}, \ldots, s_{p}}^{K}\left(z_{2}, \ldots, z_{p}\right) .
$$

La seconde somme faisant intervenir $\left(k_{2}-1\right)^{\ell}$ est à peine plus compliquée. En développant le terme $\left(k_{2}-1\right)^{\ell}$ par le théorème binomial et en remplaçant directement dans la somme, on obtient en effet :

$$
\begin{aligned}
-\sum_{\ell=0}^{s_{1}} & \frac{a_{1, \ell}\left(s_{1}, z_{1}\right)}{\left(1-z_{1}\right)^{s_{1}+1} z_{1}} \sum_{m=0}^{\ell}\left(\begin{array}{c}
\ell \\
m
\end{array}\right)(-1)^{\ell-m} \operatorname{La}_{s_{2}-m, s_{3}, \ldots, s_{p}}^{K}\left(z_{1} z_{2}, z_{3}, \ldots, z_{p}\right) \\
& -\sum_{\ell=0}^{s_{1}} \frac{a_{2, \ell}\left(s_{1}, z_{1}\right)}{\left(1-z_{1}\right)^{s_{1}+1}} \sum_{m=0}^{\ell}\left(\begin{array}{c}
\ell \\
m
\end{array}\right)(-1)^{\ell-m} \operatorname{La}_{s_{2}-m, s_{3}, \ldots, s_{p}}^{K}\left(z_{2}, z_{3}, \ldots, z_{p}\right),
\end{aligned}
$$

ce qui termine la démonstration.

6.2. Démonstration du théorème 6.1. - On remarque que le lemme 3 exprime un polylogarithme de profondeur $p$ à l'aide de polylogarithmes de profondeur $p-1$, ce qui ouvre la porte à une démonstration du théorème 6.1 par récurrence.

Pour $p=1$, le théorème est vrai, comme le montre la remarque 1) qui suit son énoncé.

On suppose que l'on sait décomposer les polylogarithmes de profondeur $\leq p-1$ (avec $p-1 \geq 1$ ) de la manière prévue par le théorème. Soit maintenant $s_{1}, \ldots, s_{p}$ une suite quelconque d'entiers, avec au moins un $s_{j} \leq 0$ : notons $q+1$ le plus petit indice $\geq 1$ tel que $s_{q+1} \leq 0$. Pour simplifier, on 
note $s_{q+1}=-s$ avec $s \geq 0$. On doit distinguer trois cas : $q=0,1 \leq q \leq p-2$ et $q=p-1$.

Le cas $q=0$. - Notons que pour tout entier $t \geq 0$, on a

$$
\sum_{k=\ell}^{\infty} k^{t} z^{k}=\left(z \frac{\mathrm{d}}{\mathrm{d} z}\right)^{t}\left(\frac{z^{\ell}}{1-z}\right)=\frac{z^{\ell} Q_{t}(\ell, z)}{(1-z)^{t+1}}
$$

avec $Q_{t}(\ell, z) \in \mathbb{Z}[\ell, z]$ de degré $s$ en $\ell$ et $z$. On pose donc

$$
Q_{t}(\ell, z)=\sum_{j=0}^{s} q_{j, s}(z) \ell^{j}
$$

On a alors

$$
\begin{gathered}
\operatorname{La}_{s_{1}, s_{2}, \ldots, s_{p}}\left(z_{1}, z_{2}, \ldots, z_{p}\right)=\sum_{k_{2} \geq \cdots \geq k_{p} \geq 1}\left(\frac{z_{2}^{k_{2}} \cdots z_{p}^{k_{p}}}{k_{2}^{s_{2}} \cdots k_{p}^{s_{p}}} \sum_{k_{1}=k_{2}}^{\infty} k_{1}^{s} z_{1}^{k_{1}}\right) \\
=\frac{1}{\left(1-z_{1}\right)^{s+1}} \sum_{k_{2} \geq \cdots \geq k_{p} \geq 1} Q_{s}\left(k_{2}, z_{1}\right) \frac{\left(z_{1} z_{2}\right)^{k_{2}} z_{3}^{k_{3}} \cdots z_{p}^{k_{p}}}{k_{2}^{s_{2}} k_{3}^{s_{3}} \cdots k_{p}^{s_{p}}} \\
=\frac{1}{\left(1-z_{1}\right)^{s+1}} \sum_{j=0}^{s} q_{j, s}\left(z_{1}\right) \mathrm{La}_{s_{2}-j, s_{3}, \ldots, s_{p}}\left(z_{1} z_{2}, z_{3}, \ldots, z_{p}\right) .
\end{gathered}
$$

Comme on n'a finalement que des «La » de profondeur $p-1$, l'hypothèse de récurrence s'applique.

Le cas $1 \leq q \leq p-2$. - On applique le lemme 3 de telle sorte que

$$
\begin{aligned}
\operatorname{La}_{s_{1}, s_{2}, \ldots, s_{p}}\left(z_{1}, z_{2}, \ldots, z_{p}\right) \\
=\sum_{k_{1} \geq \cdots \geq k_{q} \geq 1} \frac{z_{1}^{k_{1}} \cdots z_{q}^{k_{q}}}{k_{1}^{s_{1}} \cdots k_{q}^{s_{q}}} \mathrm{La}_{-s, s_{q+2}, \ldots, s_{p}}^{k_{q}}\left(z_{q+1}, z_{q+2}, \ldots, z_{p}\right) \\
=\sum_{k_{1} \geq \cdots \geq k_{q} \geq 1} \frac{z_{1}^{k_{1}} \cdots z_{q}^{k_{q}}}{k_{1}^{s_{1}} \cdots k_{q}^{s_{q}}} P_{s}\left(k_{q}, z_{q+1}\right) \mathrm{La}_{s_{q+2}, \ldots, s_{p}}^{k_{q}}\left(z_{q+2}, \ldots, z_{p}\right)
\end{aligned}
$$

TOME $136-2008-\mathrm{N}^{\circ} 1$ 


$$
\begin{aligned}
& -\sum_{\ell=0}^{s} \frac{a_{1, \ell}\left(s, z_{q+1}\right)}{\left(1-z_{q+1}\right)^{s+1} z_{q+1}} \\
& \times \sum_{m=0}^{\ell}\left((-1)^{\ell-m}\left(\begin{array}{c}
\ell \\
m
\end{array}\right) \sum_{k_{1} \geq \cdots \geq k_{q} \geq 1} \frac{z_{1}^{k_{1}} \cdots z_{q}^{k_{q}}}{k_{1}^{s_{1}} \cdots k_{q}^{s_{q}}}\right. \\
& \left.\quad \times \operatorname{La}_{s_{q+2}-m, s_{q+3}, \ldots, s_{p}}^{k_{q}}\left(z_{q+1} z_{q+2}, z_{q+3}, \ldots, z_{p}\right)\right) \\
& -\sum_{\ell=0}^{s} \frac{a_{2, \ell}\left(s, z_{q+1}\right)}{\left(1-z_{q+1}\right)^{s+1}} \\
& \quad \times \sum_{m=0}^{\ell}\left(( - 1 ) ^ { \ell - m } \left(\begin{array}{c}
\ell \\
m
\end{array} \sum_{k_{1} \geq \cdots \geq k_{q} \geq 1} \frac{z_{1}^{k_{1}} \ldots z_{q}^{k_{q}}}{k_{1}^{s_{1}} \cdots k_{q}^{s_{q}}}\right.\right. \\
& \left.\times \operatorname{La}_{s_{q+2}-m, s_{q+3}, \ldots, s_{p}}^{k_{q}}\left(z_{q+2}, z_{q+3}, \ldots, z_{p}\right)\right) .
\end{aligned}
$$

Il est facile de traiter les séries (6.4) et (6.5) puisqu'elles valent respectivment

$$
\begin{aligned}
& \mathrm{La}_{s_{1}, \ldots, s_{q}, s_{q+2}-m, s_{q+3}, \ldots, s_{p}}\left(z_{1}, \ldots, z_{q}, z_{q+1} z_{q+2}, \ldots, z_{p}\right) \text { et } \\
& \mathrm{La}_{s_{1}, \ldots, s_{q}, s_{q+2}-m, s_{q+3}, \ldots, s_{p}}\left(z_{1}, \ldots, z_{q}, z_{q+2}, \ldots, z_{p}\right),
\end{aligned}
$$

qui sont de profondeur $p-1$ : on peut donc leur appliquer l'hypothèse de récurrence.

Reste la série sur la ligne (6.3) : on utilise de nouveau la forme développée (6.2) de $P_{s}(K, z)$ pour en obtenir l'expression alternative

$$
\begin{aligned}
& \sum_{\ell=0}^{s} \sum_{k_{1} \geq \cdots \geq k_{q} \geq 1} \frac{z_{1}^{k_{1}} \cdots z_{q}^{k_{q}}}{k_{1}^{s_{1}} \cdots k_{q}^{s_{q}}} \frac{z_{q+1}^{k_{q}} a_{1, \ell}\left(s, z_{q+1}\right)+a_{2, \ell}\left(s, z_{q+1}\right)}{k_{q}^{-\ell}\left(1-z_{q+1}\right)^{s+1}} \\
& \times \operatorname{La}_{s_{q+2}, \ldots, s_{p}}^{k_{q}}\left(z_{q+2}, \ldots, z_{p}\right) \\
& =\frac{1}{\left(1-z_{q+1}\right)^{s+1}}
\end{aligned}
$$

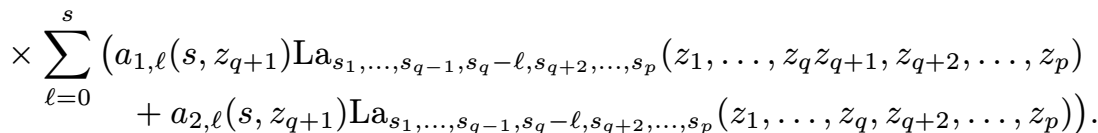

Comme on a maintenant affaire à une combinaison linéaire de « La » de profondeur $p-1$, l'hypothèse de récurrence s'applique. 
Le cas $q=p-1$. - On a

$$
\begin{aligned}
& \operatorname{La}_{s_{1}, s_{2}, \ldots, s_{p}}\left(z_{1}, z_{2}, \ldots, z_{p}\right) \sum_{k_{1} \geq \cdots \geq k_{p-1} \geq 1} \frac{z_{1}^{k_{1}} \cdots z_{q}^{k_{p-1}}}{k_{1}^{s_{1}} \cdots k_{p-1}^{s_{p-1}}} \mathrm{La}_{-s}^{k_{p-1}}\left(z_{p}\right) \\
&=\sum_{k_{1} \geq \cdots \geq k_{p-1} \geq 1} \frac{z_{1}^{k_{1}} \cdots z_{q}^{k_{q}}}{k_{1}^{s_{1}} \cdots k_{q}^{s_{q}}} P_{s}\left(k_{p-1}, z_{p}\right) \\
&=\sum_{\ell=0}^{s} \sum_{k_{1} \geq \cdots \geq k_{p-1} \geq 1} \frac{z_{1}^{k_{1}} \cdots z_{q}^{k_{q}}}{k_{1}^{s_{1}} \cdots k_{q}^{s_{q}}} \frac{z_{p}^{k_{p-1}} a_{1, \ell}\left(s, z_{p}\right)+a_{2, \ell}\left(s, z_{p}\right)}{k_{q}^{-\ell}\left(1-z_{p}\right)^{s+1}} \\
&=\frac{1}{\left(1-z_{p}\right)^{s+1}} \sum_{\ell=0}^{s}\left(a_{1, \ell}\left(s, z_{p}\right) \operatorname{La}_{s_{1}, \ldots, s_{p-2}, s_{p-2}-\ell}\left(z_{1}, \ldots, z_{p-2}, z_{p-1} z_{p}\right)\right. \\
&\left.+a_{1, \ell}\left(s, z_{p}\right) \mathrm{La}_{s_{1}, \ldots, s_{p-2}, s_{p-2}-\ell}\left(z_{1}, \ldots, z_{p-2}, z_{p-1}\right)\right) .
\end{aligned}
$$

On peut de nouveau appliquer l'hypothèse de récurrence, ce qui termine la preuve du théorème 6.1 .

\section{Démonstration du théorème 1.5}

Pour démontrer le théorème 1.5, nous devons régulariser les polyzêtas divergents intervenant dans la décomposition d'une brique. La régularisation qui s'impose ici est la régularisation dite shuffle des polyzêtas basée sur l'étude du comportement asymptotique des polylogarithmes lorsque $z$ tend vers 1 .

7.1. Régularisation « $\mathbf{s h}$ » analytique. - Dans [29, cor. 2.5], Racinet caractérise, suivant les travaux de Boutet de Monvel, le comportement asymptotique des polylogarithmes lorsque $z$ tend vers 1 .

ThÉORÈme 7.1. - Pour tous entiers strictement positifs $s_{1}, \ldots, s_{p}$, la fonction $\operatorname{Li}_{s_{1}, \ldots, s_{p}}(z)$ admet, lorsque $z$ tend vers 1 avec $|z|<1$, un développement asymptotique du type

$$
\operatorname{Li}_{s_{1}, \ldots, s_{p}}(z)=Q_{s_{1}, \ldots, s_{p}}(\log (1-z))+\mathcal{O}\left((1-z)^{\varepsilon}\right)
$$

avec $Q_{s_{1}, \ldots, s_{p}} \in \mathbb{C}[t]$ et $\varepsilon \in \mathbb{R}_{+}^{*}$.

On note $\zeta^{\amalg}\left(s_{1}, \ldots, s_{p}\right)$ la valeur régularisée de $\zeta\left(s_{1}, \ldots, s_{p}\right)$ pour $s_{1}=1$ obtenue en posant $\zeta^{\mathrm{I}}\left(s_{1}, \ldots, s_{p}\right)=Q(0)$, i.e. le terme constant du polynôme $Q$. Si $s_{1} \geq 2$, on a bien sûr $\zeta^{\mathrm{m}}\left(s_{1}, \ldots, s_{p}\right)=\zeta\left(s_{1}, \ldots, s_{p}\right)$.

Pour implémenter de façon effective notre algorithme de décomposition, il faut calculer les polyzêtas régularisés $\zeta^{\mathrm{W}}\left(s_{1}, \ldots, s_{p}\right)$ en fonction des polyzêtas classiques. Le paragraphe suivant montre comment procéder. 
7.2. Régularisation « $\mathbf{s h} »$ combinatoire. - La régularisation m que nous venons de définir conserve la symétrie «ш » vérifiée par les polyzêtas convergents. Soit $A=\{\mathbf{0}, \mathbf{1}\}$ un alphabet. On note $A^{*}$ l'ensemble des mots finis sur cet alphabet, et $A_{c}$ l'ensemble des mots de $A^{*}$ commençant par $\mathbf{0}$ et se terminant par $\mathbf{1}$. On note $\zeta$ l'application de $A_{c}$ dans $\mathbb{R}$ définie, pour tous $r \geq 1$ et $s_{1}, \ldots, s_{r} \geq 1$ avec $s_{1} \geq 2$, par

$$
\zeta\left(\mathbf{0}^{s_{1}-1} \mathbf{1 0}^{s_{2}-1} \mathbf{1} \ldots \mathbf{0}^{s_{r}-1} \mathbf{1}\right)=\zeta\left(s_{1}, \ldots, s_{r}\right) .
$$

Le produit de battage (ou shuffle) sur $A$ se définit par récurrence sur la longueur des mots par

$$
a \boldsymbol{b} \amalg c \boldsymbol{d}=a(\boldsymbol{b} \amalg c \boldsymbol{d})+c(a \boldsymbol{b} \amalg \boldsymbol{d}),
$$

pour tous $\boldsymbol{b}, \boldsymbol{d} \in A^{*}$ et $a, c \in A$.

On démontre en utilisant l'écriture intégrale des polyzêtas la relation de symétrie dite shuffle: Pour tous $\boldsymbol{u}, \boldsymbol{v} \in A_{c}$ on a

$$
\zeta(\boldsymbol{u}) \zeta(\boldsymbol{v})=\zeta(\boldsymbol{u} \amalg \boldsymbol{v}) .
$$

On renvoie par exemple à [9] ou [42] pour plus de détails.

On note $A_{0}$ l'ensemble des mots de $A^{*}$ se terminant par 1. On peut donner un sens aux polyzêtas sur $A_{0}$ en utilisant la relation (7.1) et en supposant que celle-ci est encore vérifiée pour tout mot de $A_{0}$, ce qui est le cas de la régularisation $\zeta^{\mathrm{II}}$ ci-dessus. On note encore $\zeta^{\mathrm{II}}$ le polyzêta étendu à $A_{0}$.

Pour tout mot $\underline{s}=s_{1} \cdots s_{r} \in A_{c}, r \geq 1, s_{i} \in A$, on a

$$
\mathbf{1} \amalg \mathbf{1}^{i} \underline{s}=(i+1) \mathbf{1}^{i+1} \underline{s}+\mathbf{1}^{i} s_{1}\left[\mathbf{1} \amalg \underline{s}^{>1}\right],
$$

où $\underline{s}^{>1}=s_{2} \cdots s_{r}$. En appliquant $\zeta^{\text {II }}$, on obtient

$$
\zeta^{\mathrm{II}}(\mathbf{1}) \zeta^{\mathrm{m}}\left(\mathbf{1}^{i} \underline{s}\right)=(i+1) \zeta^{\mathrm{m}}\left(\mathbf{1}^{i+1} \underline{s}\right)+\zeta^{\mathrm{m}}\left(\mathbf{1}^{i} s_{1}\left[\mathbf{1} \amalg \underline{s}^{>1}\right]\right) .
$$

Il est donc possible de calculer $\zeta^{\mathrm{m}}\left(\mathbf{1}^{i+1} \underline{s}\right)$ par récurrence sur le nombre de $\mathbf{1}$. Pour cela, il suffit de fixer une valeur à $\zeta^{\mathrm{m}}(\mathbf{1})$.

Pour obtenir une régularisation combinatoire qui coïncide avec la régularisation analytique définie au paragraphe précédent, on doit poser $\zeta^{\mathrm{m}}(1)=0$. En effet, un simple calcul donne $\operatorname{Li}_{1}(z)=-\log (1-z)$. La formule (7.2) permet alors le calcul explicite et algorithmique des polyzêtas divergents régularisés.

7.3. Énoncés. - Dans cette partie, et dans toute la suite, on pose pour $j \in$ $\{1, \ldots, p\}$ :

$$
D_{j}=\left(\sum_{i=1}^{j} A_{i}\left(n_{i}+1\right)\right)-j-1
$$


Lemme 4. - La série

$$
\sum_{k_{1} \geq \cdots \geq k_{p} \geq 1} \frac{P\left(k_{1}, \ldots, k_{p}\right)}{\left(k_{1}\right)_{n_{1}+1}^{A_{1}} \cdots\left(k_{p}\right)_{n_{p}+1}^{A_{p}}}
$$

converge si, et seulement si, le polynôme $P\left(X_{1}, \ldots, X_{p}\right)$ vérifie

$$
\sum_{i=1}^{j} \operatorname{deg}_{X_{i}} P \leq D_{j} \quad \text { pour tout } j \in\{1, \ldots, p\} .
$$

Remarque 6. - Lorsque $n_{1}=\cdots=n_{p}=0$ et $P=1$, ce lemme donne les conditions exactes de convergence des polyzêtas $\zeta\left(A_{1}, A_{2}, \ldots, A_{p}\right)$ lorsque les $A_{j}$ sont dans $\mathbb{Z}$. Elles correspondent bien aux conditions qui assurent la convergence absolue des "polyzêtas" pour des exposants complexes. Voir [24, p. 10] pour une preuve de ces conditions.

Démonstration. - On va montrer en fait que les conditions (7.3) équivalent au fait que, pour tout $B \geq 0$, la série

$$
\sum_{k_{1} \geq \cdots \geq k_{p} \geq 1} \frac{P\left(k_{1}, \ldots, k_{p}\right)\left(\log k_{p}\right)^{B}}{\left(k_{1}\right)_{n_{1}+1}^{A_{1}} \cdots\left(k_{p}\right)_{n_{p}+1}^{A_{p}}}
$$

converge. C'est évident pour $p=1$, puisque les conditions (7.3) se réduisent alors à $\operatorname{deg}_{X_{1}} P \leq A_{1}\left(n_{1}+1\right)-2$. Supposons que ce soit vrai pour $p-1$, et soit $P\left(X_{1}, \ldots, X_{p}\right) ;$ posons $\delta=\operatorname{deg}_{X_{p}} P$. Si $\delta \leq A_{p}\left(n_{p}+1\right)-1$ alors on a

$$
1 \ll \sum_{k_{p}=1}^{k_{p-1}} \frac{k_{p}^{\delta}\left(\log k_{p}\right)^{B}}{\left(k_{p}\right)_{n_{p}+1}^{A_{p}}} \ll\left(\log k_{p-1}\right)^{B+\delta}
$$

donc la convergence de (7.4) équivaut à celle de (7.4) en profondeur $p-1$. Comme justement l'équation correspondant à $j=p$ dans (7.3) se déduit des autres (puisqu'on a supposé $\operatorname{deg}_{X_{p}} P \leq A_{p}\left(n_{p}+1\right)-1$ ), la preuve est terminée dans ce cas. Supposons maintenant que l'on ait $\delta \geq A_{p}\left(n_{p}+1\right)$. Alors on a

$$
k_{p-1}^{\delta-A_{p}\left(n_{p}+1\right)+1} \ll \sum_{k_{p}=1}^{k_{p-1}} \frac{k_{p}^{\delta}\left(\log k_{p}\right)^{B}}{\left(k_{p}\right)_{n_{p}+1}^{A_{p}}} \ll k_{p-1}^{\delta-A_{p}\left(n_{p}+1\right)+1}\left(\log k_{p-1}\right)^{B}
$$

donc la convergence de (7.4) avec $P\left(X_{1}, \ldots, X_{p}\right)$ équivaut à celle de (7.4) avec un polynôme $\widetilde{P}\left(X_{1}, \ldots, X_{p-1}\right)$ vérifiant

- $\operatorname{deg}_{X_{i}} \widetilde{P}=\operatorname{deg}_{X_{i}} P$ pour $i \in\{1, \ldots, p-2\}$ et

- $\operatorname{deg}_{X_{p-1}} \widetilde{P}=\operatorname{deg}_{X_{p-1}} P+\operatorname{deg}_{X_{p}} P-A_{p}\left(n_{p}+1\right)+1$.

Or les conditions (7.3) pour un tel polynôme $\widetilde{P}$ équivalent justement aux conditions (7.3) pour $P$. Le lemme est donc démontré. 
On dit qu'une fonction $f$, définie sur un ouvert dont le point 1 appartient à l'adhérence, est à divergence au plus logarithmique en $z=1$ si elle admet un développement asymptotique de la forme

$$
f(z)=Q(\log (1-z))+\mathcal{O}\left((1-z)^{\varepsilon}\right)
$$

pour un certain $\varepsilon>0$ et un polynôme $Q \in \mathbb{C}[t]$. La valeur régularisée de $f$ en 1 est le coefficient constant de $Q$, c'est-à-dire $Q(0)$. Dans le cas particulier où $f$ est définie et continue en 1 , le polynôme $Q$ est constant et cette valeur régularisée est simplement $f(1)$. Le théorème 7.1 signifie que la fonction $\operatorname{Li}_{s_{1}, \ldots, s_{p}}(z)$ est à divergence au plus logarithmique en $z=1$; sa valeur régularisée en 1 est $\zeta^{\mathrm{m}}\left(s_{1}, \ldots, s_{p}\right)$.

\section{LEMME 5. - La fonction}

$$
\sum_{k_{1} \geq \cdots \geq k_{p} \geq 1} \frac{P\left(k_{1}, \ldots, k_{p}\right)}{\left(k_{1}\right)_{n_{1}+1}^{A_{1}} \cdots\left(k_{p}\right)_{n_{p}+1}^{A_{p}}} z^{-k_{1}}
$$

est à divergence au plus logarithmique en $z=1$ si, et seulement si,

$$
\sum_{i=1}^{j} \operatorname{deg}_{X_{i}} P \leq D_{j}+1 \quad \text { pour tout } j \in\{1, \ldots, p\} .
$$

La preuve de ce lemme est analogue à celle du lemme 4 ; seule l'initialisation diffère vraiment, puisque la fonction $\sum_{k \geq 1} k^{-1} z^{-k}$ à est divergence au plus logarithmique en $z=1$.

Pour démontrer le théorème 1.5, on va en fait démontrer le résultat suivant qui est plus fort.

THÉORÈmE 7.2. - Si les relations (7.6) sont satisfaites alors la valeur régularisée en 1 de la fonction (7.5) est une combinaison linéaire à coefficients rationnels en les polyzêtas régularisés $\zeta^{u}\left(s_{1}, \ldots, s_{q}\right)$ où $0 \leq q \leq p, s_{i} \geq 1$ pour $i=1, \ldots, q, \sum_{j=1}^{q} s_{j} \leq \sum_{j=1}^{p} A_{j}$. En outre, on peut calculer explicitement une telle combinaison linéaire.

7.4. Preuve du théorème 7.2. - On démontre le théorème 7.2 par récurrence sur la profondeur $p$. Quand $p=0$, ce théorème est trivial; les arguments qui suivent permettent de le démontrer pour $p=1$, mais un raisonnement direct est beaucoup plus facile dans ce cas. Supposons donc que ce théorème soit vrai en toute profondeur strictement inférieure à $p$.

Soit $P\left(X_{1}, \ldots, X_{p}\right)$ un polynôme tel que les relations (7.6) soient satisfaites. On pose

$$
R\left(X_{1}, \ldots, X_{p}\right)=\frac{P\left(X_{1}, \ldots, X_{p}\right)}{\left(X_{1}\right)_{n_{1}+1}^{A_{1}} \cdots\left(X_{p}\right)_{n_{p}+1}^{A_{p}}},
$$


et on étudie la fonction

$$
f(z)=\sum_{k_{1} \geq \cdots \geq k_{p} \geq 1} R\left(k_{1}, \ldots, k_{p}\right) z^{-k_{1}}
$$

qui est définie pour $|z|>1$ et est à divergence au plus logarithmique en $z=1$ grâce au lemme 5. On utilise le développement en éléments simples de $R$, comme au paragraphe 4.1 (dont on reprend les notations). Ceci permet d'écrire, pour $|z|>1$ :

$$
f(z)=\sum_{\varpi} C[\varpi] \sum_{k_{1} \geq \cdots \geq k_{p} \geq 1} \frac{\prod_{i \in I} k_{i}^{\widehat{s}_{i}}}{\prod_{i \in I^{\mathrm{c}}}\left(k_{i}+j_{i}\right)^{s_{i}}} z^{-k_{1}} .
$$

Dans cette formule et dans toute la suite, on note $\varpi$ un quadruplet générique

$$
\left(I,\left(s_{i}\right)_{i \in I^{\mathrm{c}}},\left(j_{i}\right)_{i \in I^{\mathrm{c}}},\left(\widehat{s}_{i}\right)_{i \in I}\right)
$$

tel que $1 \leq s_{i} \leq A_{i}$ et $0 \leq j_{i} \leq n_{i}$ pour tout $i \in I^{\mathrm{c}}$, et $0 \leq \widehat{s}_{i} \leq \widehat{A}_{i}$ pour tout $i \in I$. On pose alors

$$
C[\varpi]=C\left[\begin{array}{c}
I \\
\left(s_{i}\right) \\
\left(j_{i}\right) \\
\left(\widehat{s}_{i}\right)
\end{array}\right] .
$$

La difficulté est que ce développement en éléments simples peut faire apparaître des fonctions de $z$ dont la divergence en 1 n'est pas logarithmique. Par exemple, si $p=2, n_{1}=2, A_{1}=1, P\left(X_{1}, X_{2}\right)=\left(X_{2}\right)_{n_{2}+1}^{A_{2}} X_{2}$ alors les relations (7.6) sont satisfaites mais dans l'expression (7.7) apparaissent les sommes

$$
\sum_{k_{1} \geq k_{2} \geq 1} \frac{k_{2}}{k_{1}+j} z^{-k_{1}}
$$

pour $j \in\{0,1,2\}$, qui sont chacune à divergence non logarithmique. Une méthode pour résoudre ce problème serait de généraliser le théorème 7.2 , en autorisant des divergences non logarithmiques (c'est-à-dire des développements asymptotiques avec des termes $\left.\log ^{k}(z) /(1-z)^{\ell}\right)$. Mais cela nécessiterait une généralisation du théorème 7.1, et ne présenterait pas d'intérêt pratique. En effet, la présence de pôles en $1 /(1-z)$ nécessite de connaître aussi le coefficient de $1-z$ dans les développements asymptotiques, car leur produit contribue à la valeur en $z=1$. L'algorithme devrait donc calculer beaucoup de termes des développements asymptotiques, ce qui serait coûteux en temps et en mémoire. C'est pourquoi on procède plutôt comme suit. L'idée importante est celle de la régularisation : quand seules des divergences logarithmiques sont présentes, seul le coefficient constant du polynôme en $\log (1-z)$ intervient dans les calculs, y compris lorsqu'on doit faire des produits. 
Notons $\mathscr{E}_{0}$ l'ensemble des quadruplets $\varpi=\left(I,\left(s_{i}\right)_{i \in I^{\mathrm{c}}},\left(j_{i}\right)_{i \in I^{\mathrm{c}}},\left(\widehat{s}_{i}\right)_{i \in I}\right)$ tels que la fonction

$$
\sum_{k_{1} \geq \cdots \geq k_{p} \geq 1} \frac{\prod_{i \in I} \hat{k}_{i}^{\widehat{s_{i}}}}{\prod_{i \in I^{\mathrm{c}}}\left(k_{i}+j_{i}\right)^{s_{i}}} z^{-k_{1}}
$$

soit à divergence au plus logarithmique en $z=1$, et $\mathscr{E}_{1}$ son complémentaire.

Dans la somme (7.7), chaque élément $\varpi \in \mathscr{E}_{0}$ donne lieu à un développement asymptotique de la forme $Q_{\varpi}(\log (1-z))+\mathcal{O}\left((1-z)^{\varepsilon}\right)$ avec $\varepsilon>0$ (qu'on peut choisir indépendant de $\varpi)$ et $Q_{\varpi} \in \mathbb{C}[t]$. En regroupant d'autre part les contributions de tous les éléments $\varpi \in \mathscr{E}_{1}$, on a donc

$$
\begin{aligned}
\sum_{\varpi \in \mathscr{E}_{1}} C[\varpi] \sum_{k_{1} \geq \cdots \geq k_{p} \geq 1} & \frac{\prod_{i \in I} \widehat{k}_{i}^{s_{i}}}{\prod_{i \in I^{\mathrm{c}}}\left(k_{i}+j_{i}\right)^{s_{i}}} z^{-k_{1}} \\
& =f(z)-\sum_{\varpi \in \mathscr{E}_{0}} Q_{\varpi}(\log (1-z))+\mathcal{O}\left((1-z)^{\varepsilon}\right) .
\end{aligned}
$$

Comme $f(z)$ est à divergence au plus logarithmique, on voit que le membre de gauche l'est aussi; on va maintenant transformer ce membre de gauche en une somme du type (7.5) en profondeur $p-1$. Soit $\varpi \in \mathscr{E} 1$, avec

$$
\varpi=\left(I,\left(s_{i}\right)_{i \in I^{\mathrm{c}}},\left(j_{i}\right)_{i \in I^{\mathrm{c}}},\left(\widehat{s}_{i}\right)_{i \in I}\right) .
$$

L'hypothèse (7.6) (avec $j=1$ ) montre que l'ensemble $J$ défini au paragraphe 4.1 est inclus dans $\{2, \ldots, p\}$, donc $I$ aussi. En outre, $I$ est non vide (sinon on aurait $\varpi \in \mathscr{E}_{0}$ d'après le lemme 5$)$. Donc il existe $t \in\{2, \ldots, p\}$ tel que $t \in I$. En notant $B_{s}(X)$ le $s$-ième polynôme de Bernoulli (qui est à coefficients rationnels, et égal à $P_{s}(X-1,1)$ avec les notations du paragraphe $\left.6.1^{(7)}\right)$, on a

$$
\sum_{k_{t}=k_{t+1}}^{k_{t-1}} k_{t}^{\widehat{s}_{t}}=B_{\widehat{s_{t}}}\left(k_{t-1}+1\right)-B_{\widehat{s}_{t}}\left(k_{t+1}\right) .
$$

Cette relation permet d'écrire, en posant $\ell_{1}=k_{1}, \ldots, \ell_{t-1}=k_{t-1}, \ell_{t}=k_{t+1}$, $\ell_{p-1}=k_{p}$ :

$$
\begin{aligned}
& \sum_{k_{1} \geq \cdots \geq k_{p} \geq 1} \frac{\prod_{i \in I} k_{i}^{s_{i}}}{\prod_{i \in I^{\mathrm{c}}}\left(k_{i}+j_{i}\right)^{s_{i}}} z^{-k_{1}} \\
&=\sum_{\ell_{1} \geq \cdots \geq \ell_{p-1} \geq 1} \frac{\prod_{i \in I, i \leq t-1} \widehat{\ell}_{i}^{s_{i}} \prod_{i \in I, i \geq t+1} \ell_{i-1}^{\hat{s}_{i}}}{\prod_{i \in I^{\mathrm{c}}, i \leq t-1}\left(\ell_{i}+j_{i}\right)^{s_{i}} \prod_{i \in I^{\mathrm{c}}, i \geq t+1}\left(\ell_{i-1}+j_{i}\right)^{s_{i}}} \\
& \times\left(B_{\widehat{s_{t}}}\left(\ell_{t-1}+1\right)-B_{\widehat{s_{t}}}\left(\ell_{t}\right)\right) z^{-\ell_{1}} .
\end{aligned}
$$

(7) On peut noter que l'on utilise ici les mêmes idées que celles du paragraphe 6 sur le non-enrichissement des «La » à exposants négatifs. 
Cette somme est de la forme

$$
\sum_{\ell_{1} \geq \cdots \geq \ell_{p-1} \geq 1} R_{\varpi}\left(\ell_{1}, \ldots, \ell_{p-1}\right) z^{-\ell_{1}}
$$

pour une certaine fraction rationnelle $R_{\varpi}$ (qui dépend aussi du choix, arbitraire et fixé, de $t$ ). Le membre de gauche de (7.9) s'écrit donc

$$
\sum_{\ell_{1} \geq \cdots \geq \ell_{p-1} \geq 1} \widetilde{R}\left(\ell_{1}, \ldots, \ell_{p-1}\right) z^{-\ell_{1}},
$$

où l'on a posé

$$
\widetilde{R}\left(\ell_{1}, \ldots, \ell_{p-1}\right)=\sum_{\varpi \in \mathscr{E}_{1}} C[\varpi] R_{\varpi}\left(\ell_{1}, \ldots, \ell_{p-1}\right) .
$$

La relation (7.9) et le lemme 5 montrent que la fraction rationnelle $\widetilde{R}\left(\ell_{1}, \ldots, \ell_{p-1}\right)$ satisfait aux hypothèses du théorème 7.2 , en profondeur $p-1$. Par hypothèse de récurrence, on peut donc écrire (7.11) sous la forme $\widetilde{Q}(\log (1-z))+\mathcal{O}\left((1-z)^{\varepsilon}\right)$, où $\widetilde{Q}(0)$ est une combinaison linéaire explicite à coefficients rationnels en les polyzêtas régularisés $\zeta^{\mathrm{W}}\left(s_{1}, \ldots, s_{q}\right)$ où $0 \leq q \leq p-1, \sum_{j=1}^{q} s_{j} \leq \sum_{j=1}^{p} A_{j}$. Compte tenu de (7.9), il suffit maintenant de calculer $Q_{\varpi}(0)$ pour $\varpi \in \mathscr{E}_{0}$, et la preuve du théorème 7.2 sera terminée.

Pour cela, on décompose la somme (7.8). Tout d'abord, si $I$ est non vide alors on applique la relation (7.10) comme ci-dessus, et on est ramené à une profondeur strictement inférieure. On peut donc supposer que $I$ est vide. Il suffit alors de suivre la preuve du théorème 1.3 (voir le paragraphe 4 ) avec $z_{1}=z$, $z_{2}=\cdots=z_{p}=1$, puis d'appliquer le théorème 7.2 en profondeur $\leq p-1$. Ceci termine la preuve du théorème 7.2 .

\section{BIBLIOGRAPHIE}

[1] S. Akiyama, S. Egami \& Y. Tanigawa - «Analytic continuation of multiple zeta-functions and their values at non-positive integers », Acta Arith. 98 (2001), p. 107-116.

[2] S. Akiyama \& Y. Tanigawa - «Multiple zeta values at non-positive integers », Ramanujan J. 5 (2001), p. 327-351 (2002).

[3] R. ApÉRY - «Irrationalité de $\zeta(2)$ et $\zeta(3) »$, Astérisque 61 (1979), p. 11-13.

[4] W. N. BAILEY - Generalized hypergeometric series, Cambridge University Press, Cambridge, 1935.

[5] K. BAll \& T. Rivoal - «Irrationalité d'une infinité de valeurs de la fonction zêta aux entiers impairs », Invent. Math. 146 (2001), p. 193-207. 
[6] F. Beukers - «A note on the irrationality of $\zeta(2)$ and $\zeta(3) »$, Bull. London Math. Soc. 11 (1979), p. 268-272.

[7] F. C. S. Brown - «Périodes des espaces des modules $\bar{M}_{0, n}$ et multizêtas », C. R. Acad. Sci. Paris, Sér. I 336 (2006).

[8] P. CARTIER - «Fonctions polylogarithmes, nombres polyzêtas et groupes pro-unipotents », Astérisque 282 (2002), p. 137-173, Séminaire Bourbaki, Vol. 2000/2001.

[9] P. Colmez - Arithmétique de la fonction zêta, Ed. Éc. Polytech., Palaiseau, 2003.

[10] J. CRESSON - «Calcul Moulien », Prépublication IHÉS 06/22 (2006), 93 p.

[11] J. Cresson, S. Fischler \& T. Rivoal - « Code en GP-Pari de l'implémentation de l'algorithme décrit dans Séries hypergéométriques multiples et polyzêtas », preprint http://www.math.u-psud.fr/ fischler/algo. html.

[12] _ « Phénomènes de symétrie dans des formes linéaires en polyzêtas », J. reine angew. Math. 617 (2008), preprint arXiv:math.NT/0609744.

[13] J. ECALLE - «ARI/GARI, la dimorphie et l'arithmétique des multizêtas : un premier bilan », J. Théor. Nombres Bordeaux 15 (2003), p. 411-478.

[14] O. Espinosa \& V. H. Moll - «The evaluation of Tornheim double sums. I », J. Number Theory 116 (2006), p. 200-229.

[15] S. Fischler - «Multiple series connected to Hoffman's conjecture on multiple zeta values », J. of Algebra, à paraître.

[16] S. FischleR - «Groupes de Rhin-Viola et intégrales multiples », J. Théor. Nombres Bordeaux 15 (2003), p. 479-534.

[17] _ _ « Irrationalité de valeurs de zêta (d'après Apéry, Rivoal, ... ) », Astérisque 294 (2004), p. 27-62.

[18] S. Fischler \& T. RivoAL - «Approximants de Padé et séries hypergéométriques équilibrées », J. Math. Pures Appl. (9) 82 (2003), p. 1369-1394.

[19] A. B. Goncharov - «Multiple polylogarithms and mixed Tate motives », 2001.

[20] A. B. Goncharov \& Y. I. Manin - «Multiple $\zeta$-motives and moduli spaces $\overline{\mathcal{M}}_{0, n} »$, Compos. Math. 140 (2004), p. 1-14.

[21] M. HAtA - «A note on Beukers' integral », J. Austral. Math. Soc. Ser. A 58 (1995), p. 143-153.

[22] M. HATA - «A new irrationality measure for $\zeta(3) »$, Acta Arith. 92 (2000), p. $47-57$.

[23] C. Krattenthaler \& T. Rivoal - «Hypergéométrie et fonction zêta de Riemann », in Mem. Amer. Math. Soc., vol. 186, 2007. 
[24] _ , « An identity of Andrews, multiple integrals, and very-well-poised hypergeometric series », Ramanujan J. 13 (2007), p. 203-219.

[25] L. LEWIN - Polylogarithms and associated functions, North-Holland Publishing Co., 1981, With a foreword by A. J. Van der Poorten.

[26] H. N. Minh, M. Petitot \& J. Van Der Hoeven - «Shuffle algebra and polylogarithms », Discrete Math. 225 (2000), p. 217-230, Formal power series and algebraic combinatorics (Toronto, ON, 1998).

[27] Y. V. Nesterenko - «A few remarks on $\zeta(3)$ (en russe)», Mat. Zametki 59 (1996), p. 865-880, trad. anglaise Math. Notes 59 (1996), p. 625-636.

[28] G. RACINET - «Séries génératrices non-commutatives de polyzêtas et associateurs de Drinfeld », Thèse, Université d'Amiens, 2000.

[29] G. Racinet - «Doubles mélanges des polylogarithmes multiples aux racines de l'unité », Publ. Math. Inst. Hautes Études Sci. 95 (2002), p. 185231.

[30] C. Reutenauer - «Free Lie Algebras », in London Math. Soc. Monographs, new series, vol. 7, 1993.

[31] G. Rhin \& C. Viola - « On a permutation group related to $\zeta(2) »$, Acta Arith. 77 (1996), p. 23-56.

[32] _ « "The group structure for $\zeta(3) »$, Acta Arith. 97 (2001), p. 269-293.

[33] T. RIVOAL - «La fonction zêta de Riemann prend une infinité de valeurs irrationnelles aux entiers impairs », C. R. Acad. Sci. Paris Sér. I Math. 331 (2000), p. 267-270.

[34] L. J. SlateR - Generalized hypergeometric functions, Cambridge University Press, 1966.

[35] V. N. Sorokin - «On the measure of transcendency of the number $\pi^{2} »$, Mat. Sb. 187 (1996), p. 87-120, trad. anglaise Sb. Math., 187 (1996), p. $1819-1852$.

[36] , «Apéry's theorem », Vestnik Moskov. Univ. Ser. I Mat. Mekh. 3 (1998), p. 48-52, trad. anglaise Moscow Univ. Math. Bull. 3 (1998), p. $48-52$.

[37] _ « On the linear independence of values of generalized polylogarithms », Mat. Sb. 192 (2001), p. 139-154, trad. anglaise Sb. Math. 192 (2001), p. 1225-1239.

[38] H. M. SRIVAstava \& P. W. KARLSson - Multiple Gaussian hypergeometric series, Ellis Horwood Series : Mathematics and its Applications, Ellis Horwood Ltd., 1985.

[39] T. Terasoma - « Mixed Tate motives and multiple zeta values », Invent. Math. 149 (2002), p. 339-369. 
[40] E. A. UlanskiI - «Identities for generalized polylogarithms », Mat. Zametki 73 (2003), p. 613-624, trad. anglaise Math. Notes 73 (2003), p. 571581.

[41] D. V. VASILYEV - «Approximations of zero by linear forms in values of the Riemann zeta-function (en russe) », Doklady Nat. Acad. Sci Belarus 45 (2001), p. 36-40, trad. anglaise « On small linear forms for the values of the Riemann zeta-function at odd points », prépublication no. 1 (558), Nat. Acad. Sci. Belarus, Institute Math., Minsk (2001), 14 p.

[42] M. WALdschmidT - «Valeurs zêta multiples. Une introduction », J. Théor. Nombres Bordeaux 12 (2000), p. 581-595, Colloque International de Théorie des Nombres (Talence, 1999).

[43] M. Waldschmidt - « Twisted Hoffman algebras, Report 12/2003, Colloque Elementare und analytische Zahlentheorie, Oberwolfach », 2003.

[44] D. ZAGIER - «Values of zeta functions and their applications », in First European Congress of Mathematics, Vol. II (Paris, 1992), Progr. Math., vol. 120, Birkhäuser, 1994, p. 497-512.

[45] S. A. ZLobin - "Integrals that can be represented as linear forms of generalized polylogarithms », Mat. Zametki 71 (2002), p. 782-787, trad. anglaise Math. Notes 71 (2002), p. 711-716.

[46] _ « Expansion of multiple integrals in linear forms », Mat. Zametki 77 (2005), p. 683-706, trad. anglaise Math. Notes 77 (2005), p. 630-652.

[47] S. A. ZLOBIN - «Properties of coefficients of certain linear forms in generalized polylogarithms (en russe) », Fundamentalnaya $i$ Prikladnaya Matematika 11 (2005), p. 41-58, arXiv:math.NT/0511245.

[48] W. ZUDILIN - «Well-poised hypergeometric service for Diophantine problems of zeta values », J. Théor. Nombres Bordeaux 15 (2003), p. 593-626.

[49] _ « Arithmetic of linear forms involving odd zeta values », J. Théor. Nombres Bordeaux 16 (2004), p. 251-291.

[50] _ « Well-poised hypergeometric transformations of Euler-type multiple integrals », J. London Math. Soc. (2) 70 (2004), p. 215-230. 